\title{
Gravitational waves as a probe of the extra dimension
}

\author{
O-Kab Kwon ${ }^{*}$ and Seokcheon Lee ${ }^{\dagger}$ \\ Department of Physics, BK21 Physics Research Division, Institute of Basic Science, \\ Sungkyunkwan University, Suwon 16419, Korea \\ D. D. Tolla \\ Department of Physics, BK21 Physics Research Division, Institute of Basic Science, \\ Sungkyunkwan University, Suwon 16419, Korea University College, \\ Sungkyunkwan University, Suwon 16419, South Korea
}

(Received 3 July 2019; published 22 October 2019)

\begin{abstract}
We consider the Einstein-Hilbert action without a cosmological constant in five dimensions and implement the Kaluza-Klein (KK) reduction by compactifying the fifth direction on a circle of small but finite radius. For a nonzero compactification radius, the four-dimensional spectrum contains massless and massive KK modes. For the massive KK modes, we retain four KK tensor modes and one KK scalar mode after gauge fixing. We treat those massive KK modes as stochastic sources of gravitational waves (GWs) with characteristic dependence of the frequencies on the size of the extra dimension. Using the observational bounds on the size of the extra dimension and on the characteristic strain, we make an order estimation on the frequencies and amplitudes of the massive KK modes that can contribute to the GWs.
\end{abstract}

DOI: 10.1103/PhysRevD.100.084050

\section{INTRODUCTION}

The detection of gravitational waves (GWs) by the LIGO and Virgo Collaborations [1-9] from the merging of a pair of heavy black holes seems to be consistent with the prediction of Einstein's general relativity. The GW due to the merging of a neutron star pair has also been observed subsequently [10,11], opening new possibilities for astronomical observation and cosmological research. Shortly after general relativity was established, the idea to unify gravity and the electromagnetic force, known as the Kaluza-Klein (KK) reduction [12-16], was born. This was an innovative attempt based on the idea of the existence of extra dimensions, and later the presence of extra dimensions became an essential element in the study of unified theories, such as string or M-theories. For this reason, if one finds a way to test the existence of extra dimensions in nature, a new horizon of understanding of

\footnotetext{
*okab@skku.edu

Corresponding author.

skylee2@gmail.com

ddtolla@skku.edu

Published by the American Physical Society under the terms of the Creative Commons Attribution 4.0 International license. Further distribution of this work must maintain attribution to the author(s) and the published article's title, journal citation, and DOI. Funded by SCOAP .
}

physics will be opened. Despite many attempts, such a test has not been successful so far.

The current theoretical prediction of the $\mathrm{GW}$ is based on general relativity in four dimensions with no reference to the extra dimensions. However, if we are living in a universe with extra dimensions, there can be some remnants of the extra dimensions in the detection of the GW. The remnants may encode some information of the extra dimensions, such as the size of the extra dimension and the dynamics of fluctuation modes. Earlier attempts to explain the effect of extra dimensions on GW can be found in Refs. [17-27].

In this paper, we consider the $\mathrm{KK}$ reduction of the Einstein-Hilbert action in five dimensions without a cosmological constant, which has one compactified spatial dimension of small but finite size $L$ [28]. For nonzero $L$, the momenta of massive KK modes contain the information of the size of the extra dimension. We explicitly show that these massive modes contribute to metric fluctuations, which can have effects on the GW detections. To see the leading contribution in the small $L$ limit, we analyze the five-dimensional Einstein equation up to quadratic order in the metric fluctuations. We briefly summarize the procedure below.

The background manifold in our setting is given by $\mathcal{M}_{4} \times S^{1}$, where $\mathcal{M}_{4}$ is the four-dimensional Minkowski space [29]. Then, the five-dimensional metric is expressed as [30] 


$$
g_{p q}(x, y)=g_{p q}^{(0)}(x, y)+h_{p q}(x, y),
$$

where $g_{p q}^{(0)}$ represents the background metric, $h_{p q}$ is the metric fluctuation, and $x, y$ are the coordinates for the fourth and fifth dimensions, respectively. In order to investigate the effect of the extra dimension on the four-dimensional GW, we insert Eq. (1.1) into the fivedimensional Einstein equation and keep terms up to quadratic order in $h_{p q}$. We expand the fluctuations in terms of the spherical harmonics $Y^{I}(y)$ on $S^{1}$ as $h_{p q}(x, y)=$ $\sum_{I=0}^{\infty} h_{p q}^{I}(x) Y^{I}(y)$ and then project the Einstein equations on those spherical harmonics in order to obtain the equations of motion for each $\mathrm{KK}$ mode $h_{p q}^{I}$ [31]. With an appropriate gauge choice, we show that the set of dynamical fields is composed of the massless graviton zero mode $\hat{h}_{\mu \nu}^{0}$, two massless vector zero modes $v_{\mu}^{0}$, one massless scalar zero mode $s^{0}$, the massive KK scalar modes $h^{I}$, and the massive KK graviton modes $\hat{h}_{\mu \nu}^{I}$, with $I=1,2, \ldots$ The massive KK modes, $h^{I}$ and $\hat{h}_{\mu \nu}^{I}$, behave as matter fields and play the role of the source for metric fluctuations in the equation of motion for the massless graviton $\hat{h}_{\mu \nu}^{0}$. This is given by

$$
\square \bar{h}_{\mu \nu}^{0}=-\frac{16 \pi G_{4}}{c^{4}} T_{\mu \nu},
$$

where $G_{4}$ is the four-dimensional Newton constant, and $\bar{h}_{\mu \nu}^{0}$ denotes the trace-reversed graviton mode defined as $\bar{h}_{\mu \nu}^{0} \equiv$ $\hat{h}_{\mu \nu}^{0}-\frac{1}{2} g_{\mu \nu} \hat{h}_{\rho}^{0 \rho}$ satisfying the Lorenz gauge $\nabla^{\mu} \bar{h}_{\mu \nu}^{0}=0$. The energy-momentum tensor $T_{\mu \nu}$ is built from the massive KK modes and their derivatives.

The form of the $T_{\mu \nu}$ in Eq. (1.2) is complicated and noncanonical in the sense that it includes higher derivatives. This makes the task of understanding the effect of the massive KK modes on the four-dimensional GW highly nontrivial. In order to simplify the problem, we assume that the sources of GWs from the extra dimension are stochastic. This assumption is realized by the ergodic average of $T_{\mu \nu}$ denoted by $\left\langle T_{\mu \nu}\right\rangle[32,33]$. The equation of motion for the massive KK tensor modes implies that it is traceless and transverse. Because of these properties, the ergodic average of the energy-momentum tensor $\left\langle T_{\mu \nu}\right\rangle$ is fully simplified. The resulting energy density $\rho_{\mathrm{GW}}=\left\langle T_{00}\right\rangle$ of the $\mathrm{GW}$ is given by

$$
\rho_{\mathrm{GW}} \propto \int d \ln f\left[1-\left(\frac{c I}{2 \pi f L}\right)^{2}\right] f^{3} S_{h}(f),
$$

where $I=0,1, \ldots$ denotes the index of spherical harmonics, $f$ is the frequency of the massive KK modes (but can be identified with the frequency of GWs), and $S_{h}(f)$ is the spectral density of the massive KK tensor modes. This result contains a characteristic factor

$$
C_{I}(f) \equiv 1-\left(\frac{I c}{2 \pi f L}\right)^{2}
$$

which determines the contributions of massive modes to the energy density of the GW only at the specific frequencies. From the positiveness of $C_{I}(f)$ in Eq. (1.4), we see that there exists a minimum value of the frequency in the $\mathrm{GW}$ detection. For instance, the minimum frequency is given by

$$
f_{\min } \geq 4.8 \times 10^{11}(\mathrm{~Hz}),
$$

for the upper bound on $L \leq 10^{-4} \mathrm{~m}$. The smaller the size of the extra dimension, the higher the minimum frequency. The frequency ranges for the current GW detectors are far below the minimum frequency of our model. Therefore, in order to see the effect of extra dimensions on GWs, a new generation of detectors with higher frequency ranges is required. From the various stochastic background GW observational limits [34-40], one can also estimate the amplitude of the massive $\mathrm{KK}$ modes that corresponds to this minimum frequency. We present such order estimation in Sec. III B.

This paper is organized as follows. In Sec. II, we investigate the formalism of the effective four-dimensional gravitational waves from the KK reduction of the fivedimensional theory to the effective four-dimensional gravity theory. The four-dimensional massless graviton obtains the source from the massive modes. We show how to calculate this source term in the stochastic method in Sec. III. We conclude in Sec. IV.

\section{DIMENSIONAL REDUCTION WITH MASSIVE KK MODES}

We consider the KK reduction of the five-dimensional Einstein-Hilbert action without a cosmological constant to obtain the four-dimensional gravity theory. The KK reduction involves compactification of one spatial coordinate on a circle of radius $L$. For a finite $L$, the compactification results in four-dimensional KK towers, which are the tensor modes $h_{\mu \nu}^{I}$, the vector modes $v_{\mu}^{I}$, and scalar modes $s^{I}$, with $I=0,1,2, \ldots$. The masses of these KK modes are proportional to $\left(\frac{I}{L}\right)$. In the $L \rightarrow 0$ limit, all massive KK modes $(I \neq 0)$ are decoupled from the zero modes $\left(h_{\mu \nu}^{0}, v_{\mu}^{0}, s^{0}\right)$. However, if $L$ is small but not zero, there exist nontrivial couplings among the massless and a few lower massive KK modes. In this paper, with an appropriate gauge choice, we get rid of the KK massive vector modes and investigate the couplings of the massless $\mathrm{KK}$ modes to the remaining massive KK modes. The result is the four-dimensional linearized Einstein equation without a cosmological constant, but with the energy-momentum tensor determined by the massive KK modes. In this section, we present the detailed procedures. 
In this paper, we consider the five-dimensional EinsteinHilbert action without a cosmological constant,

$$
S=\frac{1}{16 \pi G_{5}} \int d^{4} x d y \sqrt{-\hat{g}} \hat{R}
$$

where $y$ denotes the coordinate of the extra dimension with $S^{1}$ geometry. The five-dimensional Einstein equation is

$$
\hat{R}_{p q}-\frac{1}{2} \hat{g}_{p q} \hat{R}=0
$$

In order to implement the KK reduction including massive KK modes, we introduce a metric fluctuation $\delta \hat{g}_{p q}=h_{p q}$ as

$$
\hat{g}_{p q}=g_{p q}+h_{p q},
$$

where $g_{p q}$ is the background metric. We plug Eq. (2.3) into Eq. (2.2) and expand the latter up to quadratic order in the fluctuations. Since the background metric $g_{p q}$ is flat, the perturbed Einstein equation becomes

$\delta \hat{R}_{p q}-\frac{1}{2}\left(g_{p q} g^{r s} \delta \hat{R}_{r s}+g^{r s} h_{p q} \delta \hat{R}_{r s}+g_{p q} \delta \hat{g}^{r s} \delta \hat{R}_{r s}\right)=0$.

Inserting $\delta \hat{g}^{p q}=-h^{p q}+h^{p r} h_{r}^{q}$ with $h^{p q} \equiv g^{p r} g^{q s} h_{r s}$ into Eq. (2.4), we obtain the equations for the fluctuations up to quadratic order in $h_{p q}$,

$$
\begin{gathered}
\nabla^{r} \nabla_{p} h_{r q}+\nabla^{r} \nabla_{q} h_{r p}-\nabla^{2} h_{p q}-\nabla_{q} \nabla_{p} h \\
-g_{p q}\left(\nabla^{r} \nabla^{s} h_{r s}-\nabla^{2} h\right)+Q_{p q}=0,
\end{gathered}
$$

with

$$
\begin{gathered}
Q_{p q}=-\nabla_{r}\left(h^{r s}\left(\nabla_{p} h_{s q}+\nabla_{q} h_{s p}-\nabla_{s} h_{p q}\right)\right) \\
+\frac{1}{2} \nabla_{q} h^{r s} \nabla_{p} h_{r s}+h^{r s} \nabla_{q} \nabla_{p} h_{r s} \\
+\frac{1}{2} \nabla^{r} h_{s}^{s}\left(\nabla_{p} h_{r q}+\nabla_{q} h_{r p}-\nabla_{r} h_{p q}\right)+\nabla^{r} h_{q}^{s} \nabla_{r} h_{s p} \\
-\nabla^{r} h^{s}{ }_{q} \nabla_{s} h_{p r}+\frac{1}{2} g_{p q} \nabla_{r}\left(h^{r s}\left(2 \nabla^{t} h_{s t}-\nabla_{s} h_{t}^{t}\right)\right) \\
-\frac{3}{4} g_{p q} \nabla^{t} h^{r s} \nabla_{t} h_{r s}+\frac{1}{2} g_{p q} \nabla^{r} h^{s t} \nabla_{s} h_{t r}-\frac{1}{2} g_{p q} h^{r s} \nabla^{2} h_{r s} \\
-\frac{1}{4} g_{p q} \nabla^{r} h^{s}{ }_{s}\left(2 \nabla^{s} h_{r s}-\nabla_{r} h_{t}^{t}\right) \\
+\frac{1}{2} g_{p q} h^{r s}\left(\nabla^{t} \nabla_{r} h_{t s}+\nabla^{t} \nabla_{s} h_{t r}-\nabla^{2} h_{r s}\right. \\
\left.-\nabla_{r} \nabla_{s} h_{t}^{t}\right)+h_{p q} \nabla^{2} h_{r}^{r}-h_{p q} \nabla_{r} \nabla_{s} h^{r s},
\end{gathered}
$$

where $Q_{p q}$ stands for terms that are quadratic in the fluctuations. Our goal in this section is to implement the
KK reduction and obtain the four-dimensional linearized Einstein equation with a source from the five-dimensional equation in Eq. (2.5). The resulting equations contain information on the size of extra dimensions only if we take into account the massive KK modes.

The line element of the flat background metric with the compactified fifth direction has the form

$$
d s^{2}=\hat{g}_{p q} d x^{p} d x^{q}=\eta_{\mu \nu} d x^{\mu} d x^{\nu}+d y^{2},
$$

where $y=L \psi$ with $0 \leq \psi<2 \pi$. The following procedure is identical to the Scherk-Schwarz formalism, which reduces $\hat{d}$-dimensional gravity to $d=\hat{d}$-1-dimensional effective theory [41]. Using the metric in Eq. (2.8), we split Eq. (2.5) into $(\mu, \nu),(\mu, 5)$, and $(5,5)$ components to obtain the equations of motion for the tensor, vector, and scalar fields. See the Appendix A. Since the fifth direction is compactified, one can expand the fluctuations as

$$
\begin{aligned}
& h_{\mu \nu}(x, \psi)=h_{\mu \nu}^{I}(x) Y^{I}(\psi), \\
& h^{\mu}{ }_{\mu}(x, \psi)=h^{I}(x) Y^{I}(\psi), \\
& h_{\mu 5}(x, \psi)=v_{\mu}^{0}(x) Y^{0}+v_{\mu}^{I}(x) \nabla_{5} Y^{I}(\psi), \\
& h_{55}(x, \psi)=s^{I}(x) Y^{I}(\psi),
\end{aligned}
$$

where $Y^{I}$ 's with $I=0,1, \ldots$ are the spherical harmonics on $S^{1}$, which satisfy the eigenequation $\nabla_{5}^{2} Y^{I}=\frac{\partial^{2}}{\partial y^{2}} Y^{I}=$ $\frac{1}{L^{2}} \frac{\partial^{2}}{\partial \psi^{2}} Y^{I}=\Lambda^{I} Y^{I}$ with $\Lambda^{I}=-\frac{I^{2}}{L^{2}}$. In order to obtain consistent gauge transformations for the four-dimensional fields, we use $\nabla_{5} Y^{I}$ instead of $Y^{I}$, in the expansion of $h_{\mu 5}$. We normalize the spherical harmonics as

$$
Y^{I}(\psi)=\sqrt{2} \cos (I \psi)
$$

so that they obey the orthonormal condition

$$
\frac{1}{2 \pi} \int_{0}^{2 \pi} d \psi Y^{I}(\psi) Y^{J}(\psi)= \begin{cases}2 & (I=J=0) \\ \delta^{I J} & (I, J>0) .\end{cases}
$$

\section{A. Gauge fixing}

Under an infinitesimal coordinate transformation $x^{\prime p}=x^{p}-\xi^{p}$, the metric fluctuation transforms as

$$
\delta_{\xi} h_{p q}=\nabla_{p} \xi_{q}+\nabla_{q} \xi_{p} .
$$

Expanding the gauge function in terms of the spherical harmonics as

$$
\begin{aligned}
& \xi_{\mu}(x, y)=\xi_{\mu}^{I}(x) Y^{I}(y), \\
& \xi_{5}(x, y)=\xi^{0}(x) Y^{0}+\xi^{I}(x) \nabla_{5} Y^{I}(y),
\end{aligned}
$$

and using Eq. (2.9) in Eq. (2.11), we obtain 


$$
\begin{aligned}
\delta_{\xi} h_{\mu \nu}^{I}(x) & =\nabla_{\mu} \xi_{\nu}^{I}(x)+\nabla_{\nu} \xi_{\mu}^{I}(x), \\
\delta_{\xi} v_{\mu}^{0} & =\nabla_{\mu} \xi^{0}, \quad \delta_{\xi} v_{\mu}^{I}(x)=\nabla_{\mu} \xi^{I}(x)+\xi_{\mu}^{I}(x), \quad(I \neq 0), \\
\delta_{\xi} s^{I}(x) & =2 \Lambda^{I} \xi^{I}(x) .
\end{aligned}
$$

Combining the tensor, the vector, and the scalar KK modes in Eq. (2.13), we obtain the gauge-invariant massive KK tensor modes for $I \neq 0$,

$$
\hat{h}_{\mu \nu}^{I}(x)=h_{\mu \nu}^{I}(x)-\left(\nabla_{\mu} v_{\nu}^{I}(x)+\nabla_{\nu} v_{\mu}^{I}(x)\right)+\frac{1}{\Lambda^{I}} \nabla_{\mu} \nabla_{\nu} s^{I}(x) .
$$

In this paper, we fix the 5 gauge degrees of freedom (d.o.f.) for the nonzero modes as follows:

$$
v_{\mu}^{I}(x)=0, \quad s^{I}(x)=h^{I}(x),
$$

where $I \neq 0$. Then, the gauge-invariant massive KK tensor modes are reduced to

$$
\hat{h}_{\mu \nu}^{I}(x)=h_{\mu \nu}^{I}(x)+\frac{1}{\Lambda^{I}} \nabla_{\mu} \nabla_{\nu} h^{I}(x) .
$$

In the next subsection, we see that the massive KK scalar modes $h^{I}$ and tensor modes $\hat{h}_{\mu \nu}^{I}$ play the role of the matter fields in the linearized Einstein equations of the massless four-dimensional graviton mode.

In addition to the five gauge conditions in Eq. (2.15), we have five more constraints, which follow from the equations of motion for the nonzero modes. As a result, we get 5 physical d.o.f. of the massive KK modes for every $I=1,2, \ldots$. See the discussion after Eq. (2.26).

For the zero modes, we use a different set of constraints to remove unphysical d.o.f. From the gauge transformations for the zero modes in Eq. (2.13), namely, $\delta_{\xi} h_{\mu \nu}^{0}=$ $\nabla_{\mu} \xi_{\nu}^{0}+\nabla_{\nu} \xi_{\mu}^{0}, \delta_{\xi} v_{\mu}^{0}=\nabla_{\mu} \xi^{0}$ and $\delta_{\xi} s^{0}=0$, we obtain five constraints. We combine some of these gauge constraints with some of the five additional constraints, which are obtained from the equations of motion for the zero modes, to impose the transverse-traceless (TT) conditions on the four-dimensional graviton mode. In the next subsection, we introduce the four-dimensional graviton mode $\hat{h}_{\mu \nu}^{0}$ and impose the TT gauge $\nabla^{\mu} \hat{h}_{\mu \nu}^{0}=0, g^{\mu \nu} \hat{h}_{\mu \nu}^{0}=0$.

\section{B. Linearized Einstein equations}

In order to obtain the equations of motion for various $\mathrm{KK}$ modes, we split Eq. (2.5) into $(\mu, \nu),(\mu, 5),(5,5)$ components and use the expansions in terms of the spherical harmonics given in Eq. (2.9). Then, the equations of motion for the Ith KK modes are obtained by projecting on $Y^{I}$ or $\nabla^{5} Y^{I}$ [42]. In particular, the equations of motion for the zero modes $(I=0)$ are given by

$$
\begin{gathered}
\nabla^{\rho} \nabla_{\mu} h_{\rho \nu}^{0}+\nabla^{\rho} \nabla_{\nu} h_{\rho \mu}^{0}-\square h_{\mu \nu}^{0}-\nabla_{\mu} \nabla_{\nu}\left(h^{0}+s^{0}\right) \\
-g_{\mu \nu}\left(\nabla^{\rho} \nabla^{\sigma} h_{\rho \sigma}^{0}-\square\left(h^{0}+s^{0}\right)\right)+Q_{\mu \nu}^{0}=0, \\
\nabla_{\mu} \nabla^{\nu} v_{\nu}^{0}-\square v_{\mu}^{0}+Q_{\mu}^{0}=0, \\
\square h^{0}-\nabla^{\rho} \nabla^{\sigma} h_{\rho \sigma}^{0}+Q_{h}^{0}=0,
\end{gathered}
$$

where $Q_{\mu \nu}^{0}, Q_{\mu}^{0}$, and $Q_{h}^{0}$ are the projections of the quadratic terms in Eq. (2.5) on $Y^{0}$, and they are composed of the massive KK modes $h_{\mu \nu}^{I}, h^{I}(I \neq 0)$. The explicit forms of $Q_{\mu \nu}^{0}$ and $Q_{h}^{0}$ are given in Eqs. (A3) and (A9), The explicit form of $Q_{\mu}^{0}$ is omitted because the equation of motion for the vector zero mode in Eq. (2.18) is decoupled from the scalar and the tensor zero modes, which means $Q_{\mu}^{0}$ is irrelevant to obtain the equation of motion for the fourdimensional graviton. The equation of motion for the scalar zero mode $s^{0}$ is obtained by combining the trace of Eq. (2.17) with Eq. (2.19), which results in

$$
\square s^{0}+Q_{s}^{0}=0,
$$

where $Q_{s}^{0}$ is given in Eq. (A11). Now we can plug Eqs. (2.19) and (2.20) into Eq. (2.17) to obtain

$$
\begin{aligned}
& \nabla^{\rho} \nabla_{\mu} h_{\rho \nu}^{0}+\nabla^{\rho} \nabla_{\nu} h_{\rho \mu}^{0}-\square h_{\mu \nu}^{0}-\nabla_{\mu} \nabla_{\nu}\left(h^{0}+s^{0}\right) \\
& \quad-g_{\mu \nu}\left(Q_{h}^{0}+Q_{s}^{0}\right)+Q_{\mu \nu}^{0}=0 .
\end{aligned}
$$

Before we proceed further, we would like to comment on the fact that the massless KK zero modes cannot contribute to the quadratic terms in the above equations. Naively, the projection of Eq. (2.5) on $Y^{0}$ produces quadratic terms that are composed of both the massless and the massive KK modes. For instance, the equation for the massless KK tensor mode can be rewritten as

$$
\begin{aligned}
& \nabla^{\rho} \nabla_{\mu} h_{\rho \nu}^{0}+\nabla^{\rho} \nabla_{\nu} h_{\rho \mu}^{0}-\square h_{\mu \nu}^{0}-\nabla_{\mu} \nabla_{\nu}\left(h^{0}+s^{0}\right) \\
& \quad=\tilde{Q}_{\mu \nu}^{0}\left(h_{\mu \nu}^{I}, h^{I}\right)+\hat{Q}_{\mu \nu}^{0}\left(h_{\mu \nu}^{0}, s^{0}, v_{\mu}^{0}\right),
\end{aligned}
$$

where $\tilde{Q}_{\mu \nu}^{0}\left(h_{\mu \nu}^{I}, h^{I}\right)$ contains only the massive KK modes whereas $\hat{Q}_{\mu \nu}^{0}\left(h_{\mu \nu}^{0}, s^{0}, v_{\mu}^{0}\right)$ comes from the massless modes. Now, if we introduce an order parameter of small fluctuations and write the zero mode as $h_{\mu \nu}^{0}=\lambda a_{\mu \nu}^{0}+\mathcal{O}\left(\lambda^{2}\right)$, then in order for $\tilde{Q}_{\mu \nu}^{0}\left(h_{\mu \nu}^{I}, h^{I}\right)$ to be of the same order as the linear terms in Eq. (2.22), the nonzero modes must be $h_{\mu \nu}^{I}=\sqrt{\lambda} a_{\mu \nu}^{I}+\mathcal{O}(\lambda)$. Thus, $\hat{Q}_{\mu \nu}^{0}\left(h_{\mu \nu}^{0}, s^{0}, v_{\mu}^{0}\right)$ will be second order in $\lambda$ and can be dropped from the linearized equations for zero modes. For this reason, the quadratic terms in the equations of motion for the massless KK modes can contain only the massive KK modes.

We can simplify the quadratic terms in the above equations by using the equations of motion for the massive modes. As those quadratic terms are already second order 
in the fluctuations, the linear order of the massive modes is sufficient for the purpose of such simplification. After fixing the gauge as in Eq. (2.15), the linear order equations of motion for the massive modes, which are obtained by projecting Eq. (2.5) on $Y^{I}$ or $\nabla^{5} Y^{I}$, are given by

$$
\begin{gathered}
\nabla_{\mu} \nabla^{\rho} h_{\rho \nu}^{I}+\nabla_{\nu} \nabla^{\rho} h_{\rho \mu}^{I}-\left(\square+\Lambda^{I}\right) h_{\mu \nu}^{I}-2 \nabla_{\mu} \nabla_{\nu} h^{I} \\
-g_{\mu \nu}\left[\nabla^{\rho} \nabla^{\sigma} h_{\rho \sigma}^{I}-\Lambda^{I} h^{I}-2 \square h^{I}\right]=0, \\
\square h^{I}=-\Lambda^{I} h^{I}, \\
\nabla^{\rho} h_{\rho \mu}^{I}=\nabla_{\mu} h^{I} .
\end{gathered}
$$

Combining Eqs. (2.24) and (2.25), we obtain one more constraint, which is

$$
h^{I}=-\frac{1}{\Lambda^{I}} \nabla^{\mu} \nabla^{\nu} h_{\mu \nu}^{I} .
$$

The five constraints from Eqs. (2.25) and (2.26) will eliminate 5 d.o.f. among the 15 d.o.f. in $h_{p q}^{I}$. An additional 5 d.o.f. are removed by the gauge fixing in Eq. (2.15). This will leave us with 5 physical d.o.f. for the nonzero modes at each $I=1,2, \ldots$.

For the zero modes, the TT gauge, which was introduced in the previous subsection, removes 5 unphysical d.o.f. from $\hat{h}_{\mu \nu}^{0}$. We then use the remaining five constraints to remove 3 more unphysical d.o.f. from $\hat{h}_{\mu \nu}^{0}$ and 2 unphysical d.o.f. from $v_{\mu}^{0}$. As a result, we have 5 physical d.o.f. for the
TABLE I. The physical d.o.f. of five-dimensional fields and four-dimensional fields. Instead of 2 physical d.o.f. of $h_{\mu \nu}$, we choose 4 physical d.o.f. for $\hat{h}_{\mu \nu}^{I}$ by removing 2 d.o.f. of $v_{\mu}^{I}$.

\begin{tabular}{cccccc}
\hline \hline \multirow{2}{*}{ Modes } & Five-dimensional & \multicolumn{4}{c}{ Four-dimensional } \\
& fields & d.o.f. & fields & d.o.f. Mass \\
\hline \multirow{3}{*}{$I=0$} & & & $\hat{h}_{\mu \nu}^{0}$ & 2 & 0 \\
& $h_{p q}^{0}$ & 5 & $v_{\mu}^{0}$ & 2 & 0 \\
& & & $s^{0}$ & 1 & 0 \\
$I \neq 0$ & $h_{p q}^{I}$ & 5 & $\hat{h}_{\mu \nu}^{I}$ & 4 & $I / L$ \\
& & & $h^{I}$ & 1 & $I / L$ \\
\hline \hline
\end{tabular}

zero modes as well. For clarity, we summarize the physical d.o.f. for the massless and massive modes in Table I.

Now inserting Eqs. (2.24) and (2.25) into Eq. (2.23), we obtain the linearized equation for the massive $\mathrm{KK}$ tensor mode,

$$
\square h_{\mu \nu}^{I}=-\Lambda^{I} h_{\mu \nu}^{I} .
$$

The mass of these KK modes is inversely proportional to the size of the compactified fifth dimension. Thus, we can constrain this size if we can measure the mass of the KK modes. We will discuss this in Sec. III.

Finally, we can use Eqs. (2.24), (2.25), and (2.27) to simplify the quadratic terms in Eqs. (2.20) and (2.21). After the simplification, we combine Eqs. (2.20) and (2.21) to obtain the equation of motion for the four-dimensional massless graviton mode,

$$
\begin{aligned}
& L_{E} \hat{h}_{\mu \nu}^{0}-\frac{1}{4} \nabla^{\sigma} h_{\rho \nu}^{I} \nabla^{\rho} h_{\sigma \mu}^{I}+\frac{1}{4} \nabla^{\sigma} h_{\nu}^{I \rho} \nabla_{\sigma} h_{\mu \rho}^{I}+\frac{1}{8} \nabla_{\mu} h^{I \rho \sigma} \nabla_{\nu} h_{\rho \sigma}^{I}+\frac{1}{4} h^{I \rho \sigma} \nabla_{\nu} \nabla_{\mu} h_{\rho \sigma}^{I}+\frac{1}{4} h^{I \rho \sigma} \nabla_{\rho} \nabla_{\sigma} h_{\mu \nu}^{I} \\
& -\frac{1}{4} h^{I \rho \sigma}\left[\nabla_{\rho} \nabla_{\mu} h_{\sigma \nu}^{I}+\nabla_{\rho} \nabla_{\nu} h_{\sigma \mu}^{I}\right]-\frac{\Lambda^{I}}{4} h_{\nu}^{I \rho} h_{\mu \rho}^{I}+\frac{1}{4} \Lambda^{I} h^{I} h_{\mu \nu}^{I}+\frac{1}{8} \nabla_{\mu} h^{I} \nabla_{\nu} h^{I}+\frac{1}{4} h^{I} \nabla_{\mu} \nabla_{\nu} h^{I} \\
& \quad+g_{\mu \nu}\left[\frac{\Lambda^{I}}{16} h^{I \rho \sigma} h_{\rho \sigma}^{I}+\frac{1}{8} h^{I \rho \sigma} \nabla_{\rho} \nabla_{\sigma} h^{I}+\frac{1}{8} \nabla^{\rho} h^{I} \nabla_{\rho} h^{I}-\frac{\Lambda^{I}}{16} h^{I} h^{I}\right]=0,
\end{aligned}
$$

where we have defined the four-dimensional massless graviton $\hat{h}_{\mu \nu}^{0}=h_{\mu \nu}^{0}+\frac{1}{2} g_{\mu \nu} s^{0}$. Here, we introduced the Einstein operator

$$
L_{E} A_{\mu \nu}=\frac{1}{2}\left(-\square A_{\mu \nu}+\nabla^{\rho} \nabla_{\mu} A_{\rho \nu}+\nabla^{\rho} \nabla_{\nu} A_{\rho \mu}-\nabla_{\mu} \nabla_{\nu} A_{\rho}^{\rho}\right) \text {. }
$$

The result in Eq. (2.28) is the linearized Einstein equation with the source for the four-dimensional graviton. One can see that the massive KK modes become the source of the four-dimensional massless graviton. Compared to the usual four-dimensional graviton which does not contain the source for the linear order perturbation, the effective four-dimensional massless graviton obtains the source from the massive KK modes in the compactified five-dimensional universe.

We can write Eq. (2.28) more formally as

$$
L_{E} \hat{h}_{\mu \nu}^{0}=8 \pi G_{N}\left(T_{\mu \nu}\left[h_{\rho \sigma}^{I}, h^{I}\right]-\frac{1}{2} g_{\mu \nu} T\left[h_{\rho \sigma}^{I}, h^{I}\right]\right)
$$

where the energy-momentum tensor is given by 


$$
\begin{aligned}
T_{\mu \nu}\left[h_{\rho \sigma}^{I}, h^{I}\right]= & -\frac{1}{32 \pi G_{N}}\left\{\nabla^{\sigma} h_{\nu}^{I \rho} \nabla_{\sigma} h_{\mu \rho}^{I}-\nabla^{\sigma} h_{\rho \nu}^{I} \nabla^{\rho} h_{\sigma \mu}^{I}+\frac{1}{2} \nabla_{\mu} h^{I \rho \sigma} \nabla_{\nu} h_{\rho \sigma}^{I}\right. \\
& +h^{I \rho \sigma} \nabla_{\nu} \nabla_{\mu} h_{\rho \sigma}^{I}+h^{I \rho \sigma} \nabla_{\rho} \nabla_{\sigma} h_{\mu \nu}^{I}-h^{I \rho \sigma}\left[\nabla_{\rho} \nabla_{\mu} h_{\sigma \nu}^{I}+\nabla_{\rho} \nabla_{\nu} h_{\sigma \mu}^{I}\right] \\
& -\Lambda^{I} h_{\nu}^{I \rho} h_{\mu \rho}^{I}+\Lambda^{I} h^{I} h_{\mu \nu}^{I}+\frac{1}{2} \nabla_{\mu} h^{I} \nabla_{\nu} h^{I}+h^{I} \nabla_{\mu} \nabla_{\nu} h^{I} \\
& \left.-\frac{g_{\mu \nu}}{4}\left[3 \nabla^{\tau} h^{I \rho \sigma} \nabla_{\tau} h_{\rho \sigma}^{I}-2 \nabla^{\rho} h^{I \sigma \tau} \nabla_{\sigma} h_{\rho \tau}^{I}-3 \Lambda^{I} h^{I \rho \sigma} h_{\rho \sigma}^{I}+3 \nabla^{\rho} h^{I} \nabla_{\rho} h^{I}-\Lambda^{I} h^{I} h^{I}\right]\right\}
\end{aligned}
$$

We note that even though $h^{I}$ appears in the energy-momentum tensor, it is not an independent d.o.f. since it is expressed in terms of $h_{\mu \nu}^{I}$ as in Eq. (2.26).

For later convenience, we rewrite Eq. (2.31) in terms of the gauge-invariant massive KK tensor mode defined in Eq. (2.16). From Eq. (2.24), we obtain $\nabla^{\mu} \hat{h}_{\mu \nu}^{I}=0$ and $g^{\mu \nu} \hat{h}_{\mu \nu}^{I}=0$. Thus,

$$
\begin{aligned}
T_{\mu \nu}\left[\hat{h}_{\rho \sigma}^{I}, h^{I}\right]= & -\frac{1}{16 \pi G_{N}}\left\{\frac{1}{2} \hat{h}^{I \rho \sigma} \nabla_{\rho} \nabla_{\sigma} \hat{h}_{\mu \nu}^{I}-\frac{1}{2} \hat{h}^{I \rho \sigma} \nabla_{\rho} \nabla_{\mu} \hat{h}_{\sigma \nu}^{I}-\frac{1}{2} \hat{h}^{I \rho \sigma} \nabla_{\rho} \nabla_{\nu} \hat{h}_{\sigma \mu}^{I}+\frac{1}{2} \hat{h}^{I \rho \sigma} \nabla_{\nu} \nabla_{\mu} \hat{h}_{\rho \sigma}^{I}\right. \\
& +\frac{1}{2} \nabla^{\sigma} \hat{h}_{\nu}^{I \rho} \nabla_{\sigma} \hat{h}_{\mu \rho}^{I}-\frac{1}{2} \nabla^{\sigma} \hat{h}_{\rho \nu}^{I} \nabla^{\rho} \hat{h}_{\sigma \mu}^{I}+\frac{1}{4} \nabla_{\mu} \hat{h}^{I \rho \sigma} \nabla_{\nu} \hat{h}_{\rho \sigma}^{I}-\frac{\Lambda^{I}}{2} \hat{h}_{\nu}^{I \rho} \hat{h}_{\mu \rho}^{I} \\
& -\frac{1}{4 \Lambda^{I}} \nabla_{\mu} \nabla^{\rho} \nabla^{\sigma} h^{I} \nabla_{\nu} \hat{h}_{\rho \sigma}^{I}-\frac{1}{4 \Lambda^{I}} \nabla_{\nu} \nabla^{\rho} \nabla^{\sigma} h^{I} \nabla_{\mu} \hat{h}_{\rho \sigma}^{I}-\frac{1}{2 \Lambda^{I}} \nabla^{\rho} \nabla^{\sigma} h^{I} \nabla_{\rho} \nabla_{\sigma} \hat{h}_{\mu \nu}^{I} \\
& +\frac{1}{2 \Lambda^{I}} \nabla^{\rho} \nabla^{\sigma} h^{I} \nabla_{\rho} \nabla_{\mu} \hat{h}_{\sigma \nu}^{I}+\frac{1}{2 \Lambda^{I}} \nabla^{\rho} \nabla^{\sigma} h^{I} \nabla_{\rho} \nabla_{\nu} \hat{h}_{\mu \sigma}^{I}-\frac{1}{2 \Lambda^{I}} \nabla^{\rho} \nabla^{\sigma} h^{I} \nabla_{\mu} \nabla_{\nu} \hat{h}_{\rho \sigma}^{I} \\
& +\frac{1}{2 \Lambda^{I}} \Lambda^{I} \nabla_{\mu} \nabla^{\rho} h^{I} \hat{h}_{\rho \nu}^{I}+\frac{1}{2 \Lambda^{I}} \Lambda^{I} \nabla_{\nu} \nabla^{\rho} h^{I} \hat{h}_{\mu \rho}^{I}+\frac{1}{2} \Lambda^{I} h^{I} \hat{h}_{\mu \nu}^{I} \\
& +\frac{1}{4\left(\Lambda^{I}\right)^{2}} \nabla_{\mu} \nabla^{\rho} \nabla^{\sigma} h^{I} \nabla_{\nu} \nabla_{\rho} \nabla_{\sigma} h^{I}-\frac{1}{2 \Lambda^{I}} \nabla_{\mu} \nabla^{\rho} h^{I} \nabla_{\nu} \nabla_{\rho} h^{I}+\frac{1}{4} \nabla_{\mu} h^{I} \nabla_{\nu} h^{I} \\
& -\frac{1}{2} g_{\mu \nu}\left[\frac{3}{4} \nabla_{\tau} \hat{h}^{I \rho \sigma} \nabla^{\tau} \hat{h}_{\rho \sigma}^{I}-\frac{1}{2} \nabla^{\sigma} \hat{h}_{\rho \tau}^{I} \nabla^{\rho} \hat{h}_{\sigma}^{I \tau}-\frac{3 \Lambda^{I}}{4} \hat{h}^{I \rho \sigma} \hat{h}_{\rho \sigma}^{I}-\frac{1}{2 \Lambda^{I}} \nabla^{\tau} \nabla^{\rho} \nabla^{\sigma} h^{I} \nabla_{\tau} \hat{h}_{\rho \sigma}^{I}\right. \\
& +\frac{3}{2} \nabla^{\rho} \nabla^{\sigma} h^{I} \hat{h}_{\rho \sigma}^{I}+\frac{1}{4\left(\Lambda^{I}\right)^{2}} \nabla^{\tau} \nabla^{\rho} \nabla^{\sigma} h^{I} \nabla_{\tau} \nabla_{\rho} \nabla_{\sigma} h^{I}-\frac{3}{4 \Lambda^{I}} \nabla^{\rho} \nabla^{\sigma} h^{I} \nabla_{\sigma} \nabla_{\rho} h^{I} \\
& \left.\left.+\frac{3}{4} \nabla_{\rho} h^{I} \nabla^{\rho} h^{I}-\frac{\Lambda^{I}}{4} h^{I} h^{I}\right]\right\} .
\end{aligned}
$$

Even though it is redundant to rewrite the $T_{\mu \nu}$ in Eq. (2.32), this form will further simplify our calculations in the subsequent sections. For comparison, we shortly review how to obtain the effective energy-momentum tensor of the four-dimensional gravitational waves on the flat universe in Appendix B. It is important to notice that the effective energy-momentum tensor obtained there is different from the above energy-momentum tensor of our model.

\section{EFFECT OF THE EXTRA DIMENSION IN GW DETECTION}

In this section, we show the effect of massive KK modes on the dynamics of the four-dimensional graviton. In order to compare with the observation, we derive the analytic form of the stochastic average of the energy-momentum tensor obtained in the previous section. We perform the order estimation to put a new constraint on the size of the extra dimension. It is expected that there will be more accurate GW data available in the future, and we believe our results provide a tangible formalism on how to use the GW data to get some information about the extra dimension.

\section{A. Stochastic average of the energy-momentum tensor}

The energy in GW is described by averaging the energymomentum tensor over several wavelengths or periods. Thus, we need to average the energy-momentum tensor in our model given in Eq. (2.32), which is built from the nonzero modes $h^{I}$ and $\hat{h}_{\mu \nu}^{I}$. Inserting the plane wave solution $\hat{h}_{\mu \nu}^{I}=A_{\mu \nu}^{I} e^{i p_{\mu} x^{\mu}}$ into Eq. (2.27), we obtain 


$$
\begin{aligned}
\square \hat{h}_{\mu \nu}^{I}+\Lambda^{I} \hat{h}_{\mu \nu}^{I} & =\left(-p_{\mu} p^{\mu}+\Lambda^{I}\right) \hat{h}_{\mu \nu}^{I}=0 \rightarrow m_{\mathrm{I}}^{2} \\
& =-\frac{\Lambda^{I} \hbar^{2}}{c^{2}}=\left(\frac{I \hbar}{c L}\right)^{2},
\end{aligned}
$$

where we have used the dispersion relation

$$
\frac{\omega_{I}^{2}}{c^{2}}-\left|\vec{k}_{I}\right|^{2}=\frac{m_{I}^{2} c^{2}}{\hbar^{2}}
$$

and the definition $\Lambda^{I}=-\frac{I^{2}}{L^{2}}$. We can regard both $h^{I}$ and $\hat{h}_{\mu \nu}^{I}$ as random variables, which can be identified with a stochastic background. Thus, using the dispersion relations, we calculate the ensemble average of the energymomentum tensor. In order to perform the time average, we use the plane wave expansion of $h^{I}(\vec{x}, t)$ in terms of the Fourier transformation [32,33],

$h^{I}(\vec{x}, t)=\int \frac{d^{3} k}{(2 \pi)^{3}}\left[\tilde{B}^{I}(\vec{k}) e^{i k_{\mu} x^{\mu}}+\tilde{B}^{I *}(\vec{k}) e^{-i k_{\mu} x^{\mu}}\right]$,

where the four-vectors in the exponent are $k^{\mu}=(\omega / c, \vec{k})$, $x^{\mu}=(c t, \vec{x})$, and we have suppressed the index $I$ in $\left(\omega_{I}, \vec{k}_{I}\right)$ for simplicity. Using the dispersion relation in Eq. (3.2), we can write $d^{3} k$ as

$$
\begin{aligned}
d^{3} k=|\vec{k}|^{2} d|\vec{k}| d^{2} \hat{n} & =\left(1+\frac{c^{2}}{\omega^{2}} \Lambda^{I}\right)^{\frac{1}{2}} \frac{\omega^{2}}{c^{3}} d \omega d^{2} \hat{n} \\
& =\sqrt{1-\left(\frac{c I}{2 \pi f L}\right)^{2}}\left(\frac{2 \pi}{c}\right)^{3} f^{2} d f d^{2} \hat{n},
\end{aligned}
$$

where $d^{2} \hat{n}=-d(\cos \theta) d \phi$ denotes the integration over the solid angle and in the last step we have replaced $\omega$ by $2 \pi f$. Similarly

$k_{\mu} x^{\mu}=-\omega t+|\vec{k}| \hat{n} \cdot \vec{x}=-2 \pi f\left(t-\sqrt{1-\left(\frac{c I}{2 \pi f L}\right)^{2}} \frac{\hat{n} \cdot \vec{x}}{c}\right)$.

Using Eqs. (3.4) and (3.5), Eq. (3.3) becomes

$$
\begin{aligned}
h^{I}(\vec{x}, t)= & \int_{\epsilon}^{\infty} d f \int d^{2} \hat{n} \sqrt{1-\left(\frac{I c}{2 \pi f L}\right)^{2}} \\
& \times\left[\frac{f^{2}}{c^{3}} \tilde{B}^{I}(f, \hat{n}) e^{-2 \pi i f\left(t-\sqrt{\left.1-\left(\frac{c I}{2 \pi f L}\right)^{2} \frac{\hat{n} \cdot \vec{x}}{c}\right)}+(\text { c.c })\right]}\right. \\
\equiv & \int_{-\infty}^{\infty} d f \int d^{2} \hat{n} \sqrt{1-\left(\frac{I c}{2 \pi f L}\right)^{2}} \\
& \times \tilde{h}^{I}(f, \hat{n}) e^{-2 \pi i f\left(t-\sqrt{\left.1-\left(\frac{c l}{2 \pi f L}\right)^{2} \frac{\hat{n} \cdot \hat{x}}{c}\right)}\right.}
\end{aligned}
$$

where $\epsilon=m c^{2} /(2 \pi \hbar)$ and in the second line we used $\tilde{B}^{I *}(f, \hat{n})=\tilde{B}^{I}(-f, \hat{n})$. Here, (c.c) means the complex conjugate, and we also introduced $\tilde{h}^{I}(f, \hat{n}) \equiv \frac{f^{2}}{c^{3}} \tilde{B}^{I}(f, \hat{n})$. We emphasize that the integral range in Eq. (3.6) is not well defined for $|f|<\epsilon$ because it will produce tachyonic modes as shown in Eq. (3.2). The real integral ranges in the second equality of Eq. (3.6) are $[-\infty,-\epsilon]$ and $[\epsilon, \infty]$. However, this detail is not important because, as we will see later, the quantities we need to compare with the data are the integrands of Eqs. (3.8), (3.14), and (3.15).

From the above plane wave solution and assumption on the stochastic properties of the nonzero modes, we can replace the ensemble average of the energy-momentum tensor with the temporal average. This is given by

$$
\begin{aligned}
& \left\langle h^{I *}(\vec{x}, t) h^{I}(\vec{x}, t)\right\rangle \\
& =\int_{-\infty}^{\infty} d f \int d^{2} \hat{n} \sqrt{1-\left(\frac{I c}{2 \pi f L}\right)^{2}} \\
& \quad \times \int_{-\infty}^{\infty} d f^{\prime} \int d^{2} \hat{n}^{\prime} \sqrt{1-\left(\frac{I c}{2 \pi f^{\prime} L}\right)^{2}} \\
& \quad \times\left\langle\tilde{h}^{I *}(f, \hat{n}) \tilde{h}^{I}\left(f^{\prime}, \hat{n}^{\prime}\right)\right\rangle e^{2 \pi i f\left(t-\sqrt{1-\left(\frac{c I}{2 \pi f L}\right)^{2} \frac{2}{2} \cdot \vec{x}} \frac{\hat{c}}{c}\right.} \\
& \quad \times e^{-2 \pi i f^{\prime}\left(t-\sqrt{\left.1-\left(\frac{c I}{2 \pi f^{\prime} L}\right)^{2} \frac{\hat{n}^{\prime} \cdot \vec{x}}{c}\right)}\right.} .
\end{aligned}
$$

Note that since $h^{I}(\vec{x}, t)$ is a real mode, we could write $\left\langle h^{I *}(\vec{x}, t) h^{I}(\vec{x}, t)\right\rangle$ instead of $\left\langle h^{I}(\vec{x}, t) h^{I}(\vec{x}, t)\right\rangle$ because the former way of writing is commonly used in the literature. Now, we use the definition of the stochastic property $\left\langle\tilde{h}^{*}(f, \hat{n}) \tilde{h}\left(f^{\prime}, \hat{n}^{\prime}\right)\right\rangle \equiv \frac{1}{2} \delta\left(f-f^{\prime}\right) \frac{\delta^{2}\left(\hat{n}, \hat{n}^{\prime}\right)}{4 \pi} S_{h}(f)$. The function $S_{h}(f)$ is called the spectral density of the stochastic background $h^{I}(\vec{x}, t)$. Then, we obtain

$$
\begin{aligned}
\left\langle h^{I *}(\vec{x}, t) h^{I}(\vec{x}, t)\right\rangle & =\frac{1}{2} \int_{-\infty}^{\infty} d f \int d^{2} \hat{n}\left[1-\left(\frac{I c}{2 \pi f L}\right)^{2}\right] \frac{S_{h}(f)}{4 \pi} \\
& =\int_{\epsilon}^{\infty} d f\left[1-\left(\frac{I c}{2 \pi f L}\right)^{2}\right] S_{h}(f),
\end{aligned}
$$

where we have used $S_{h}(-f)=S_{h}(f)$ in the second line.

One can repeat the same process for $\hat{h}_{\rho \sigma}^{I}$ to obtain

$$
\begin{aligned}
\hat{h}_{\rho \sigma}^{I}(\vec{x}, t)= & \int \frac{d^{3} k}{(2 \pi)^{3}}\left[\mathcal{A}_{\rho \sigma}^{I}(\vec{k}) e^{i k_{\mu} x^{\mu}}+\mathcal{A}_{\rho \sigma}^{I *}(\vec{k}) e^{-i k_{\mu} x^{\mu}}\right] \\
= & \int_{-\infty}^{\infty} d f \int d^{2} \hat{n} \sqrt{1-\left(\frac{I c}{2 \pi f L}\right)^{2}} \\
& \times \tilde{h}_{\rho \sigma}^{I}(f, \hat{n}) e^{-2 \pi i f\left(t-\sqrt{\left.1-\left(\frac{c l}{2 \pi L}\right)^{2} \frac{2}{2} \cdot \vec{x}\right)}\right.}
\end{aligned}
$$

where we have defined $\tilde{h}_{\rho \sigma}^{I}(f, \hat{n}) \equiv \frac{f^{2}}{c^{3}} \mathcal{A}_{\rho \sigma}(f, \hat{n})$. If we adopt the stochastic background of the GW generated from $h_{\mu \nu}^{I}$, then 


$$
\tilde{h}_{\mu \nu}^{I}(f, \hat{n})=\sum_{A} \tilde{h}_{A}^{I}(f, \hat{n}) \epsilon_{\mu \nu}^{A}
$$

where $\epsilon_{\mu \nu}^{A}$ are the polarization tensors and $A$ labels the polarization modes $(A=1,2, \ldots, 4)$ as shown in Table I. If one assumes that the stochastic backgrounds of GWs are stationary, then the ergodic average becomes

$$
\begin{aligned}
& \left\langle\tilde{h}_{\rho \sigma}^{I *}(f, \hat{n}) \tilde{h}^{\rho \sigma I}\left(f^{\prime}, \hat{n}^{\prime}\right)\right\rangle \\
& \quad \equiv \sum_{A} \epsilon_{\rho \sigma}^{A} \sum_{A^{\prime}} \epsilon^{A^{\prime} \rho \sigma}\left\langle\tilde{h}_{A}^{I *}(f, \hat{n}) \tilde{h}_{A^{\prime}}^{I}\left(f^{\prime}, \hat{n}^{\prime}\right)\right\rangle \\
& \quad=\sum_{A} \epsilon_{\rho \sigma}^{A} \epsilon^{A \rho \sigma} \delta\left(f-f^{\prime}\right) \frac{\delta^{2}\left(\hat{n}, \hat{n}^{\prime}\right)}{4 \pi} \frac{1}{2} S_{h^{I}}(f),
\end{aligned}
$$

where we have used $\left\langle\tilde{h}_{A}^{I *}(f, \hat{n}) \tilde{h}_{A^{\prime}}^{I}\left(f^{\prime}, \hat{n}^{\prime}\right)\right\rangle \equiv$ $\delta\left(f-f^{\prime}\right) \frac{\delta^{2}\left(\hat{n}, \hat{n}^{\prime}\right)}{4 \pi} \delta_{A A^{\prime}} \frac{1}{2} S_{h^{I}}(f)$. Now using Eqs. (3.9) and (3.11), the average for the tensor modes becomes

$$
\begin{aligned}
& \left\langle\hat{h}_{\rho \sigma}(\vec{x}, t) \hat{h}^{\rho \sigma}(\vec{x}, t)\right\rangle \\
& \quad=\sum_{A} \epsilon_{\rho \sigma}^{A} \epsilon^{A \rho \sigma} \int_{\epsilon}^{\infty} d f\left[1-\left(\frac{c I}{2 \pi f L}\right)^{2}\right] S_{h^{I}}(f) \\
& \quad=\sum_{A} \epsilon_{\rho \sigma}^{A} \epsilon^{A \rho \sigma} \int_{f=\epsilon}^{\infty} d \ln f\left[1-\left(\frac{c I}{2 \pi f L}\right)^{2}\right] f S_{h^{I}}(f) \\
& \quad \equiv \frac{\sum_{A} \epsilon_{\rho \sigma}^{A} \epsilon^{A \rho \sigma}}{2} \int_{f=\epsilon}^{\infty}(d \ln f) h_{c}^{2}(f),
\end{aligned}
$$

where the definition of characteristic strain is $h_{c}=\sqrt{2\left(1-(c I / 2 \pi f L)^{2}\right) f S_{h^{I}}}$. For $I=0$, we recover the usual four-dimensional characteristic strain $\sqrt{2 f S_{h^{I}}}$. The dimension of $S_{h^{I}}$ is $\left[\mathrm{Hz}^{-1}\right]$, and $h_{c}$ is dimensionless.

Finally, we can calculate $\left\langle T_{\mu \nu}\right\rangle$ using the above stochastic properties of $h^{I}$ and $\hat{h}_{\mu \nu}^{I}$. The energy-momentum tensor for our model is given in Eq. (2.32), and it looks very complicated. However, once we substitute the above plane wave solutions for the massive scalar and tensor modes, most of the terms are either vanishing because of the transverse condition $\nabla^{\mu} \hat{h}_{\mu \nu}^{I} \rightarrow k^{\mu} \hat{h}_{\mu \nu}^{I}=0$ or they cancel each other because any two contracted covariant derivatives of the form $\left\langle\nabla^{\rho}\left(\nabla \cdots h^{I}\right) \nabla_{\rho}\left(\nabla \cdots h_{\mu \nu}^{I}\right)\right\rangle$ become $\Lambda^{I}\left\langle\left(\nabla \cdots h^{I}\right)\left(\nabla \cdots h_{\mu \nu}^{I}\right)\right\rangle$, where the ellipses denote multiple covariant derivatives. As a result, we get a very remarkable simplification, and only two terms in Eq. (2.32) survive,

$$
\begin{aligned}
\left\langle T_{\mu \nu}\right\rangle= & -\frac{c^{4}}{16 \pi G}\left\langle\frac{1}{2} \hat{h}^{I \rho \sigma}\left(x^{\alpha}\right) \nabla_{\mu} \nabla_{\nu} \hat{h}_{\rho \sigma}^{I}\left(x^{\alpha}\right)\right. \\
& \left.+\frac{1}{4} \nabla_{\mu} \hat{h}^{I \rho \sigma}\left(x^{\alpha}\right) \nabla_{\nu} \hat{h}_{\rho \sigma}^{I}\left(x^{\alpha}\right)\right\rangle \\
= & \frac{c^{4}}{16 \pi G} \frac{\sum_{A} \epsilon_{\rho \sigma}^{A} \epsilon^{A \rho \sigma}}{4} \int_{f=\epsilon}^{\infty} d \ln f k_{\mu} k_{\nu} \\
& \times\left[1-\left(\frac{c I}{2 \pi f L}\right)^{2}\right] f S_{h^{I}}(f) .
\end{aligned}
$$

Thus, the energy density of the GW $\left(\rho_{\mathrm{gw}}=\left\langle T_{00}\right\rangle\right)$ is given by

$$
\begin{aligned}
\rho_{\mathrm{gw}}= & \int_{f=\epsilon}^{\infty} d \ln f \frac{d \rho_{\mathrm{gw}}}{d \ln f}=\frac{c^{2} \pi}{4 G} \frac{\sum_{A} \epsilon_{\rho \sigma}^{A} \epsilon^{A \rho \sigma}}{4} \\
& \times \int_{f=\epsilon}^{\infty} d \ln f\left[1-\left(\frac{c I}{2 \pi f L}\right)^{2}\right] f^{3} S_{h^{I}}(f),
\end{aligned}
$$

where the first step amounts to expressing the energy density of the GW as an integral over $d \ln f$ of some spectral density and the second step is substituting $\left\langle T_{00}\right\rangle$ from Eq. (3.13). One can also define the logarithmic derivative of the energy density contrast of the GW as

$$
\begin{aligned}
\Omega_{\mathrm{gw}}(f) \equiv & \frac{1}{\rho_{\mathrm{cr}}} \frac{d \rho_{\mathrm{gw}}}{d \ln f}=\frac{2 \pi^{2}}{3 H_{0}^{2}} \frac{\sum_{A} \epsilon_{\rho \sigma}^{A} \epsilon^{A \rho \sigma}}{4} \\
& \times\left[1-\left(\frac{c I}{2 \pi f L}\right)^{2}\right] f^{3} S_{h^{I}}(f) \\
= & \frac{2 \pi^{2}}{3 H_{0}^{2}} f^{2} h_{c}(f)^{2}=\frac{8 \pi^{2}}{3 H_{0}^{2}} f^{4}|\tilde{h}(f)|^{2},
\end{aligned}
$$

where $\rho_{\text {cr }} \equiv \frac{3 c^{2}}{8 \pi G} H_{0}^{2}$ is the critical energy density, $H_{0}$ is the present value of the Hubble constant, and $|\tilde{h}(f)|=$ $h_{c}(f) /(2 f)$ is the so-called frequency-domain strain. We have also used the fact that $\sum_{A} \epsilon_{\rho \sigma}^{A} \epsilon^{A \rho \sigma}=8$ in the second equality because the four massive tensor modes $\hat{h}_{\mu \nu}^{I}$ contribute to the sources of the stochastic background GW.

\section{B. Order estimations}

In this section, we elaborate on the observational applications of our model by using both $\mathrm{GW}$ experiments and particle physics ones.

\section{Gravitational wave application}

One of the main properties of our model is the existence of the lower limit of the frequency which can contribute as a source of the four-dimensional GWs. From Eq. (3.15) we notice that we first need to make sure $1-\frac{I c}{2 \pi f L}$ is positive. This means that the KK modes below a specific frequency cannot contribute as the source of the effective fourdimensional massless gravitational waves. Thus, we obtain 
TABLE II. Various observational upper limits on $\Omega_{\mathrm{gw}}$ of the stochastic GWs. The LIGO and Virgo results at different frequencies give different values of $\Omega_{\mathrm{gw}}(f)$, and thus the $h_{c}$ 's are different.

\begin{tabular}{lccccc}
\hline \hline$\Omega_{\mathrm{gw}}(f)$ & $f(\mathrm{~Hz})$ & Experiments & $h_{c}$ & $\tilde{h}$ & Reference \\
\hline 0.044 & $3 \times 10^{-4}$ & Cassini & $2 \times 10^{-15}$ & $8.32 \times 10^{-11}$ & {$[34]$} \\
$3.88 \times 10^{17}$ & $0.035-0.830$ & TOBA & $7.71 \times 10^{-9}$ & $7.71 \times 10^{-8}$ & {$[35]$} \\
$1.2 \times 10^{8}$ & $0.05-0.1$ & Seismic & $1.29 \times 10^{-13}$ & $8.61 \times 10^{-13}$ & {$[36]$} \\
$0.035-0.15$ & $0.005-0.3$ & Earth's ring & $5.51 \times 10^{-19}$ & $9.19 \times 10^{-19}$ & {$[37]$} \\
$5.6 \times 10^{-6}$ & $41.5-169$ & LIGO and Virgo & $1.91 \times 10^{-23}$ & $9.08 \times 10^{-26}$ & {$[38]$} \\
$1.8 \times 10^{-4}$ & $170-600$ & Apollo Seismic & $2.95 \times 10^{-23}$ & $3.83 \times 10^{-26}$ & \\
$1.2 \times 10^{5}$ & $0.1-1$ & Planck & $2.93 \times 10^{-16}$ & $1.47 \times 10^{-16}$ & {$[39]$} \\
$10^{-15}$ & $0.1-1$ & $2.68 \times 10^{-26}$ & $1.34 \times 10^{-26}$ & {$[40]$} \\
\hline \hline
\end{tabular}

the lower limit on the frequency $f_{\min }$ for the first massive mode $(I=1)$ for a given upper bound on the size of the extra dimension $L_{\max }$,

$$
f_{\min } \geq \frac{c}{2 \pi L_{\max }} .
$$

There have been various upper limits on the size of the extra dimension depending on the methods of measurements [43]. One way to put the limit on the $L_{\max }$ is to use the deviations of the Newtonian gravitational law, and this gives a value of around $100 \mu \mathrm{m}$ [43]. In this case, we can put the lower limit on the frequency as $\mathcal{O}\left(10^{11}\right) \mathrm{Hz}$. Another method is based on the Randall-Sundrum (RS) model which proposes the $1 / \mathrm{TeV}$ (i.e., $10^{-18} \mathrm{~m}$ ) scale warped extra dimension in order to explain the hierarchy of the electroweak scale [44]. Thus, the RS model estimates the lowest value of the frequency to be $\mathcal{O}\left(10^{25}\right) \mathrm{Hz}$. The smaller the size of the extra dimension, the higher the frequency of the modes that can contribute as the source of the massless four-dimensional GWs. Therefore, we propose high-frequency detectors in order to measure the very small extra dimension by using the GWs.

Next, we use the observational limit on the magnitude of the dimensionless energy density in GWs, $\Omega_{\mathrm{gw}}(f)$, to constrain the magnitude of the characteristic strain $h_{c}$ by using Eq. (3.15). There are various limits on the $\Omega_{\mathrm{gw}}(f)$ at fixed frequencies from various observations [34-40]. We show these in Table II. If we adopt the Planck result [40] and use the fact that $\frac{\Omega_{\mathrm{gw}}\left(f_{\mathrm{P}}\right)}{f_{\mathrm{Pl}}^{2}}=\frac{\Omega_{\mathrm{gw}}\left(f_{\mathrm{KK}}\right)}{f_{\mathrm{KK}}^{2}}$, then we can estimate the magnitude of $\tilde{h}$ as

$$
\begin{aligned}
\tilde{h}=\frac{h_{c}}{2 f} & =\sqrt{\frac{3 \Omega_{\mathrm{gw}}}{8}} \frac{H_{0}}{\pi f^{2}}=\frac{h_{c}(\mathrm{Pl})}{2 f(\mathrm{KK})} \\
& \leq \frac{3.78 \times 10^{-25}}{2 \times 10^{11}} \simeq 10^{-36}\left(\mathrm{~Hz}^{-1}\right) .
\end{aligned}
$$

\section{Particle physics application}

Due to our detailed calculation for the KK reduction with the finite size of the extra dimension, one can also estimate the size of the extra dimension by using the results of particle physics observations. From the definition of masses of KK modes, one obtains $L=\frac{I \hbar}{m_{I} c}$. We can find $L$ from the stable radion mass bound. In our model, $h^{I}$ in Eq. (2.15) corresponds to this radion. Given a stabilization mechanism, the radion mass can, in principle, be calculated. The required ansatz is that the radion fluctuation about the RS background solves the linearized Einstein equations. Then, we incorporate the backreaction of the bulk scalar vacuum expectation value into the metric. Treating the backreaction as a perturbation about the RS solution, the mass bound is given by [45-47]

$$
\mathcal{O}(10 \mathrm{GeV}) \leq m_{I} \leq \mathcal{O}(1 \mathrm{TeV}) .
$$

Thus, if we adopt this constraint to the mass-length relation in Eq. (3.2), then we obtain

$$
10^{-19} \mathrm{~m} \leq L \leq 10^{-17} \mathrm{~m}
$$

\section{CONCLUSIONS}

In order to examine the effect of the extra dimension in our observational four-dimensional phenomena, one needs to consider the small but finite size of the extra dimensions. The conventional KK reduction, where only the massless KK zero modes are kept to obtain four-dimensional gravity theory, fails to provide evidence on higher-dimensional gravity models. In the limit of vanishing size of extra dimensions, neglecting the massive KK modes is feasible, whereas, for the small but finite size of the extra dimensions, the massive KK modes can have measurable effects on the four-dimensional gravity theory. In this paper, we investigate the effective four-dimensional gravity theory obtained from the KK reduction of the Einstein-Hilbert action without the cosmological constant in a compactified five-dimensional manifold.

In our model, the KK reduction produces sets of both massless and massive dynamical fields. The massless fields are composed of a tensor mode and a vector mode, each with 2 dynamical d.o.f., and a scalar mode with 1 dynamical d.o.f. The massive fields also have 5 d.o.f., and we choose four massive tensor modes and one massive 
scalar mode. The massive modes act as the matter field and become the sources of the massless fields in the GW equation. In particular, the energy-momentum tensor in the equation of motion of the effective four-dimensional massless graviton is composed of the massive tensor and scalar KK modes.

In Ref. [23], the authors claim that there exists one longitudinal massive graviton mode in the KK reduction of five-dimensional gravity theory. Their model is quite similar to ours; however, we treat all the massive KK modes as the stochastic background of the massless graviton modes. Thus, the four-dimensional effective graviton is massless, as we have shown in Sec. II. Our result is also consistent with the previous works [41,48-50].

From the observational upper limit on the size of the extra dimension, we will be able to obtain a lower limit on the frequency of the GW. A higher minimum frequency is required to probe the very small size of the extra dimension. Also, from the various stochastic background GW observations, we estimate the amplitude of the massive KK modes $\tilde{h}_{\rho \sigma} \sim 10^{-31} \mathrm{~Hz}^{-1}$. Therefore, we propose that high frequency and very sensitive experiments of GWs are required in order to investigate the information on the five-dimensional compactified extra dimension. However, this high sensitivity might be improved if one considers higher extra dimensional models. As a side remark, we also obtain a bound on the size of the extra dimension as $10^{-19} \mathrm{~m} \leq L \leq 10^{-17} \mathrm{~m}$, from the mass bounds on the KK modes. If the observations of the particle physics provide more stringent bounds on the mass of the KK modes, this can narrow the frequency searching window.

\section{ACKNOWLEDGMENTS}

We would like to thank Hyun Seok Yang and Sang-Heon Yi for helpful discussions. O. K. acknowledges the hospitality at APCTP during the program " $100+4$ General Relativity and Beyond," where part of this work was completed. O. K., S. L., and D. T. are supported by Basic Science Research Program through the National Research Foundation of Korea (NRF) funded by the Ministry of Science, ICT and Future Planning [Grants No. NRF-2017R1D1A1A09000951, No. NRF-2019R1A6A1A10073079 (O. K.), No. NRF2017R1A2B4011168 (S. L.), and No. NRF2017R1D1A1B03032523 (D. T.), respectively].

\section{APPENDIX A: DECOMPOSITION OF QUADRATIC ORDER EQUATIONS AND HARMONIC EXPANSIONS}

We decompose Eqs. (2.5) into the $(\mu, \nu),(\mu, 5)$, and $(5,5)$ components. As a result, we obtain the following set of equations.

(i) $(\mu, \nu)$ components:

Choosing the free indices $(p, q)$ in Eq. (2.5) to be $(\mu, \nu)$, we obtain

$$
\begin{aligned}
& \nabla^{\rho} \nabla_{\mu} h_{\rho \nu}+\nabla^{\rho} \nabla_{\nu} h_{\rho \mu}+\nabla^{a} \nabla_{\mu} h_{a \nu}+\nabla^{a} \nabla_{\nu} h_{a \mu}-\left(\nabla_{\rho}^{\rho}+\nabla_{a}^{a}\right) h_{\mu \nu}-\nabla_{\mu} \nabla_{\nu}\left(h_{\rho}^{\rho}+h_{a}^{a}\right) \\
& \quad-g_{\mu \nu}\left(\nabla^{\rho} \nabla^{\sigma} h_{\rho \sigma}+2 \nabla^{\rho} \nabla^{a} h_{\rho a}+\nabla^{a} \nabla^{b} h_{a b}-\left(\nabla^{\rho} \nabla_{\rho}+\nabla^{a} \nabla_{a}\right)\left(h_{\sigma}^{\sigma}+h_{b}^{b}\right)\right)+Q_{\mu \nu}=0,
\end{aligned}
$$

where $Q_{\mu \nu}$ is the quadratic part in Eq. (2.6). Since we are considering a flat background metric, we note that $\nabla_{\rho} \nabla_{\sigma}=\nabla_{\sigma} \nabla_{\rho}$. In order to obtain the equation of motion for the tensor zero mode $h_{\mu \nu}^{0}$, we expand the fluctuation $h_{p q}$ in Eq. (A1) in terms of the spherical harmonic in Eq. (2.9) and project the resulting equations on $Y^{0}$. Using the gauge fixing (2.15), we obtain

$$
\nabla^{\rho} \nabla_{\mu} h_{\rho \nu}^{0}+\nabla^{\rho} \nabla_{\nu} h_{\rho \mu}^{0}-\square h_{\mu \nu}^{0}-\nabla_{\mu} \nabla_{\nu}\left(h^{0}+s^{0}\right)-g_{\mu \nu}\left(\nabla^{\rho} \nabla^{\sigma} h_{\rho \sigma}^{0}-\square\left(h^{0}+s^{0}\right)\right)+Q_{\mu \nu}^{0}=0,
$$

where

$$
\begin{aligned}
Q_{\mu \nu}^{0}= & -\frac{1}{2} \nabla_{\rho} h^{I \rho \sigma}\left(\nabla_{\mu} h_{\sigma \nu}^{I}+\nabla_{\nu} h_{\sigma \mu}^{I}\right)+\frac{1}{4} \nabla_{\mu} h^{I \rho \sigma} \nabla_{\nu} h_{\rho \sigma}^{I}+\frac{1}{2} \nabla_{\rho} h^{I \rho \sigma} \nabla_{\sigma} h_{\mu \nu}^{I}-\frac{1}{2} \nabla^{\sigma} h_{\rho \nu}^{I} \nabla^{\rho} h_{\sigma \mu}^{I} \\
& +\frac{1}{2} \nabla^{\sigma} h_{\nu}^{I \rho} \nabla_{\sigma} h_{\mu \rho}^{I}-\frac{1}{2} h^{I \rho \sigma} \nabla_{\rho} \nabla_{\mu} h_{\sigma \nu}^{I}-\frac{1}{2} h^{I \rho \sigma} \nabla_{\rho} \nabla_{\nu} h_{\sigma \mu}^{I}+\frac{1}{2} h^{I \rho \sigma} \nabla_{\nu} \nabla_{\mu} h_{\rho \sigma}^{I} \\
& -\frac{\Lambda^{I}}{2} h_{\nu}^{I \rho} h_{\mu \rho}^{I}+\frac{1}{4} \nabla^{\rho} h^{I}\left(\nabla_{\mu} h_{\rho \nu}^{I}+\nabla_{\nu} h_{\rho \mu}^{I}\right)-\frac{1}{4} \nabla^{\rho} h^{I} \nabla_{\rho} h_{\mu \nu}^{I}+\frac{1}{2} h_{\mu \nu}^{I} \square h^{I}+\frac{3 \Lambda^{I}}{4} h^{I} h_{\mu \nu}^{I} \\
& -\frac{1}{4} \nabla^{\rho} h^{I} \nabla_{\rho} h_{\mu \nu}^{I}+\frac{1}{4} \nabla^{\rho} h^{I}\left(\nabla_{\mu} h_{\rho \nu}^{I}+\nabla_{\nu} h_{\rho \mu}^{I}\right)+\frac{1}{2} h_{\mu \nu}^{I} \square h^{I}+\frac{\Lambda^{I}}{4} h_{\mu \nu}^{I} h^{I} \\
& +\frac{1}{4} \nabla_{\mu} h^{I} \nabla_{\nu} h^{I}+\frac{1}{2} h^{I} \nabla_{\mu} \nabla_{\nu} h^{I}+\frac{1}{2} h_{\rho \sigma}^{I} \nabla^{\rho} \nabla^{\sigma} h_{\mu \nu}^{I}-\frac{1}{2} \nabla^{\rho} \nabla^{\sigma} h_{\rho \sigma}^{I} h_{\mu \nu}^{I} \\
& +g_{\mu \nu}\left[\frac{1}{2} \nabla_{\rho} h^{I \rho \sigma} \nabla^{\tau} h_{\sigma \tau}^{I}-\frac{3}{8} \nabla^{\tau} h^{I \rho \sigma} \nabla_{\tau} h_{\rho \sigma}^{I}+\frac{1}{4} \nabla^{\sigma} h^{I \rho \tau} \nabla_{\rho} h_{\sigma \tau}^{I}-\frac{1}{2} h^{I \rho \sigma} \square h_{\rho \sigma}^{I}-\frac{\Lambda^{I}}{8} h^{I \rho \sigma} h_{\rho \sigma}^{I}\right.
\end{aligned}
$$




$$
\begin{aligned}
& +h^{I \rho \sigma} \nabla_{\rho} \nabla^{\tau} h_{\sigma \tau}^{I}-\frac{1}{2} \nabla_{\rho} h^{I \rho \sigma} \nabla_{\sigma} h^{I}-\frac{1}{2} h^{I \rho \sigma} \nabla_{\rho} \nabla_{\sigma} h^{I}+\frac{1}{8} \nabla^{\rho} h^{I} \nabla_{\rho} h^{I}-\frac{\Lambda^{I}}{8} h^{I} h^{I} \\
& -\frac{1}{2} \nabla_{\rho} h^{I \rho \sigma} \nabla_{\sigma} h^{I}-\frac{1}{2} h^{I \rho \sigma} \nabla_{\rho} \nabla_{\sigma} h^{I}+\frac{1}{4} \nabla^{\rho} h^{I} \nabla_{\rho} h^{I}-\frac{\Lambda^{I}}{4} h^{I} h^{I} \\
& \left.-\frac{1}{4} \nabla^{\rho} h^{I} \nabla_{\rho} h^{I}-\frac{1}{2} h^{I} \square h^{I}\right] .
\end{aligned}
$$

For later convenience, we take the trace of the above equation,

$$
\begin{aligned}
\square & h^{0}+\frac{3}{2} \square s^{0}-\nabla^{\rho} \nabla^{\sigma} h_{\rho \sigma}^{0}+\frac{1}{2} \nabla_{\rho} h^{I \rho \sigma} \nabla^{\tau} h_{\sigma \tau}^{I}-\frac{3}{8} \nabla^{\tau} h^{I \rho \sigma} \nabla_{\tau} h_{\rho \sigma}^{I}+\frac{1}{4} \nabla^{\sigma} h^{I \rho \tau} \nabla_{\rho} h_{\sigma \tau}^{I} \\
& +\frac{1}{2} h^{I \rho \sigma} \nabla_{\rho} \nabla^{\tau} h_{\sigma \tau}^{I}+h^{I \rho \sigma} \nabla^{\tau} \nabla_{\rho} h_{\sigma \tau}^{I}-\frac{3}{4} h^{I \rho \sigma} \square h_{\rho \sigma}^{I}-\frac{1}{2} \Lambda^{I} h^{I \rho \sigma} h_{\rho \sigma}^{I}-\frac{1}{2} \nabla_{\rho} h^{I \rho \sigma} \nabla_{\sigma} h^{I} \\
& -\frac{3}{4} h^{I \rho \sigma} \nabla_{\rho} \nabla_{\sigma} h^{I}-\frac{1}{4} \nabla^{\rho} \nabla^{\sigma} h_{\rho \sigma}^{I} h^{I}+\frac{1}{8} \nabla^{\rho} h^{I} \nabla_{\rho} h^{I}+\frac{1}{4} h^{I} \square h^{I}+\frac{1}{8} \Lambda^{I} h^{I} h^{I}-\frac{3}{4} \nabla_{\rho} h^{I \rho \sigma} \nabla_{\sigma} h^{I} \\
& -h^{I \rho \sigma} \nabla_{\rho} \nabla_{\sigma} h^{I}+\frac{3}{8} \nabla^{\rho} h^{I} \nabla_{\rho} h^{I}+\frac{1}{4} h^{I} \square h^{I}-\frac{3 \Lambda^{I}}{8} h^{I} h^{I}-\frac{3}{8} \nabla^{\rho} h^{I} \nabla_{\rho} h^{I}-\frac{3}{4} h^{I} \square h^{I}=0 .
\end{aligned}
$$

(ii) $(\mu, 5)$ components:

The $\left(\mu, a^{\prime}=5\right)$ components of Eq. (2.5) are given by

$$
\begin{aligned}
& \nabla^{\rho} \nabla_{\mu} h_{\rho a^{\prime}}+\nabla^{\rho} \nabla_{a^{\prime}} h_{\rho \mu}+\nabla^{a} \nabla_{\mu} h_{a a^{\prime}}+\nabla^{a} \nabla_{a^{\prime}} h_{a \mu}-\left(\nabla_{\rho}^{\rho}+\nabla_{a}^{a}\right) h_{\mu a^{\prime}}-\nabla_{\mu} \nabla_{a^{\prime}}\left(h_{\rho}^{\rho}+h_{a}^{a}\right) \\
& \quad-g_{\mu a^{\prime}}\left(\nabla^{\rho} \nabla^{\sigma} h_{\rho \sigma}+2 \nabla^{\rho} \nabla^{a} h_{\rho a}+\nabla^{a} \nabla^{b} h_{a b}-\left(\nabla^{\rho} \nabla_{\rho}+\nabla^{a} \nabla_{a}\right)\left(h_{\sigma}^{\sigma}+h_{b}^{b}\right)\right)+Q_{\mu 5}=0 .
\end{aligned}
$$

Projecting on $Y^{0}$ and $\nabla^{5} Y^{I}$, we obtain the equation of motion for the vector zero mode and some constraint equations for $h_{\mu \nu}^{I}$,

$$
\nabla_{\mu} \nabla^{\nu} v_{\nu}^{0}-\square v_{\mu}^{0}+Q_{\mu}^{0}=0, \quad \nabla^{\nu} h_{\mu \nu}^{I}-\nabla_{\mu} h^{I}+Q_{\mu}^{I}=0,
$$

where we have used the gauge fixing in Eq. (2.15), and $\left(Q_{\mu}^{0}, Q_{\mu}^{I}\right)$ are quadratic terms, whose explicit forms are not needed for what we want to accomplish in this paper.

(iii) $(5,5)$ components:

The $\left(a^{\prime}=5, b^{\prime}=5\right)$ components of Eq. (2.5) are

$$
\begin{aligned}
& \nabla^{\rho} \nabla_{a^{\prime}} h_{\rho b^{\prime}}+\nabla^{\rho} \nabla_{b^{\prime}} h_{\rho a^{\prime}}+\nabla^{a} \nabla_{a^{\prime}} h_{a b^{\prime}}+\nabla^{a} \nabla_{b^{\prime}} h_{a a^{\prime}}-\left(\nabla_{\rho}^{\rho}+\nabla_{a}^{a}\right) h_{a^{\prime} b^{\prime}}-\nabla_{a^{\prime}} \nabla_{b^{\prime}}\left(h_{\rho}^{\rho}+h_{a}^{a}\right) \\
& -g_{a^{\prime} b^{\prime}}\left(\nabla^{\rho} \nabla^{\sigma} h_{\rho \sigma}+2 \nabla^{\rho} \nabla^{a} h_{\rho a}+\nabla^{a} \nabla^{b} h_{a b}-\left(\nabla^{\rho} \nabla_{\rho}+\nabla^{a} \nabla_{a}\right)\left(h_{\sigma}^{\sigma}+h_{b}^{b}\right)\right)+Q_{a^{\prime} b^{\prime}}=0 .
\end{aligned}
$$

Again, we expand Eq. (A7) in terms of the spherical harmonic (2.9) and project the resulting equation on $Y^{0}$. With our gauge fixing condition (2.15), we obtain

$$
\square h^{0}-\nabla^{\rho} \nabla^{\sigma} h_{\rho \sigma}^{0}+Q_{h}^{0}=0,
$$

where

$$
\begin{aligned}
Q_{h}^{0}= & \frac{1}{2} \nabla_{\rho} h^{I \rho \sigma} \nabla^{\tau} h_{\sigma \tau}^{I}-\frac{3}{8} \nabla^{\tau} h^{I \rho \sigma} \nabla_{\tau} h_{\rho \sigma}^{I}+\frac{1}{4} \nabla^{\sigma} h^{I \rho \tau} \nabla_{\rho} h_{\sigma \tau}^{I} \\
& +h^{I \rho \sigma} \nabla_{\rho} \nabla^{\tau} h_{\sigma \tau}^{I}-\frac{1}{2} h^{I \rho \sigma} \square h_{\rho \sigma}^{I}+\frac{\Lambda^{I}}{8} h^{I \rho \sigma} h_{\rho \sigma}^{I}-\frac{1}{2} \nabla_{\rho} h^{I \rho \sigma} \nabla_{\sigma} h^{I}-\frac{1}{2} h^{I \rho \sigma} \nabla_{\rho} \nabla_{\sigma} h^{I} \\
& +\frac{1}{8} \nabla^{\rho} h^{I} \nabla_{\rho} h^{I}-\frac{\Lambda^{I}}{8} h^{I} h^{I}-\frac{1}{2} h^{I} \nabla^{\rho} \nabla^{\sigma} h_{\rho \sigma}^{I}+\frac{1}{2} h^{I} \square h^{I}
\end{aligned}
$$


Combining Eqs. (A4) and (A8), we obtain the equation of motion for zero modes $s^{0}$,

$$
\square s^{0}+Q_{s}^{0}=0,
$$

where

$$
\begin{aligned}
Q_{s}^{0} \equiv & -\left(-\frac{1}{3} h^{I \rho \sigma} \nabla_{\rho} \nabla^{\tau} h_{\sigma \tau}^{I}+\frac{1}{6} h^{I \rho \sigma} \square h_{\rho \sigma}^{I}+\frac{5 \Lambda^{I}}{12} h^{I \rho \sigma} h_{\rho \sigma}^{I}+\frac{1}{2} \nabla^{\rho} h^{I} \nabla^{\sigma} h_{\rho \sigma}^{I}+\frac{1}{6} h^{I} \nabla^{\rho} \nabla^{\sigma} h_{\rho \sigma}^{I}+\frac{1}{6} h^{I \rho \sigma} \nabla_{\rho} \nabla_{\sigma} h^{I}-\frac{1}{6} h^{I} \square h^{I}\right. \\
& -\frac{1}{6} \Lambda^{I} h^{I} h^{I}+\frac{2}{3} h^{I \rho \sigma} \nabla_{\rho} \nabla_{\sigma} h^{I}-\frac{1}{3} h^{I} \nabla^{\rho} \nabla^{\sigma} h_{\rho \sigma}^{I}-\frac{1}{4} \nabla^{\rho} h^{I} \nabla_{\rho} h^{I}-\frac{1}{6} h^{I} \square h^{I}+\frac{\Lambda^{I}}{4} h^{I} h^{I} \\
& \left.+\frac{1}{4} \nabla^{\rho} h^{I} \nabla_{\rho} h^{I}+\frac{1}{3} h^{I} \square h^{I}+\frac{1}{2} h^{I} \square h^{I}\right) .
\end{aligned}
$$

Inserting Eqs. (A8) and (A10) into Eq. (A2), we obtain

$$
\begin{aligned}
\square h_{\mu \nu}^{0}= & \nabla^{\rho} \nabla_{\mu} h_{\rho \nu}^{0}+\nabla^{\rho} \nabla_{\nu} h_{\rho \mu}^{0}-\nabla_{\mu} \nabla_{\nu}\left(h^{0}+s^{0}\right)-\frac{1}{2} \nabla_{\rho} h^{I \rho \sigma}\left(\nabla_{\mu} h_{\sigma \nu}^{I}+\nabla_{\nu} h_{\sigma \mu}^{I}\right)+\frac{1}{2} \nabla_{\rho} h^{I \rho \sigma} \nabla_{\sigma} h_{\mu \nu}^{I}+\frac{1}{4} \nabla_{\mu} h^{I \rho \sigma} \nabla_{\nu} h_{\rho \sigma}^{I} \\
& -\frac{1}{2} \nabla^{\sigma} h_{\rho \nu}^{I} \nabla^{\rho} h_{\sigma \mu}^{I}+\frac{1}{2} \nabla^{\sigma} h_{\nu}^{I \rho} \nabla_{\sigma} h_{\mu \rho}^{I}+\frac{1}{2} h^{I \rho \sigma} \nabla_{\rho} \nabla_{\sigma} h_{\mu \nu}^{I}-\frac{1}{2} \nabla^{\rho} \nabla^{\sigma} h_{\rho \sigma}^{I} h_{\mu \nu}^{I}-\frac{1}{2} h^{I \rho \sigma} \nabla_{\rho} \nabla_{\mu} h_{\sigma \nu}^{I}-\frac{1}{2} h^{I \rho \sigma} \nabla_{\rho} \nabla_{\nu} h_{\sigma \mu}^{I} \\
& +\frac{1}{2} h^{I \rho \sigma} \nabla_{\nu} \nabla_{\mu} h_{\rho \sigma}^{I}+\frac{1}{4} \nabla^{\rho} h^{I}\left(\nabla_{\mu} h_{\rho \nu}^{I}+\nabla_{\nu} h_{\rho \mu}^{I}\right)-\frac{\Lambda^{I}}{2} h_{\nu}^{I \rho} h_{\mu \rho}^{I}-\frac{1}{4} \nabla^{\rho} h^{I} \nabla_{\rho} h_{\mu \nu}^{I}+\frac{1}{2} h_{\mu \nu}^{I} \square h^{I}+\frac{3 \Lambda^{I}}{4} h^{I} h_{\mu \nu}^{I} \\
& +\frac{1}{4} \nabla^{\rho} h^{I}\left(\nabla_{\mu} h_{\rho \nu}^{I}+\nabla_{\nu} h_{\rho \mu}^{I}\right)-\frac{1}{4} \nabla_{\rho} h_{\mu \nu}^{I} \nabla^{\rho} h^{I}+\frac{1}{2} h_{\mu \nu}^{I} \square h^{I}+\frac{\Lambda^{I}}{4} h_{\mu \nu}^{I} h^{I}+\frac{1}{4} \nabla_{\mu} h^{I} \nabla_{\nu} h^{I}+\frac{1}{2} h^{I} \nabla_{\mu} \nabla_{\nu} h^{I} \\
& +g_{\mu \nu}\left(\frac{1}{6} h^{I \rho \sigma} \square h_{\rho \sigma}^{I}-\frac{1}{3} h^{I \rho \sigma} \nabla_{\rho} \nabla^{\tau} h_{\sigma \tau}^{I}+\frac{\Lambda^{I}}{6} h^{I \rho \sigma} h_{\rho \sigma}^{I}+\frac{1}{6} h^{I} \nabla^{\rho} \nabla^{\sigma} h_{\rho \sigma}^{I}+\frac{1}{6} h^{I \rho \sigma} \nabla_{\rho} \nabla_{\sigma} h^{I}-\frac{1}{6} h^{I} \square h^{I}-\frac{\Lambda^{I}}{6} h^{I} h^{I}\right. \\
& \left.+\frac{1}{6} h^{I \rho \sigma} \nabla_{\rho} \nabla_{\sigma} h^{I}+\frac{1}{6} h^{I} \nabla^{\rho} \nabla^{\sigma} h_{\rho \sigma}^{I}-\frac{1}{6} h^{I} \square h^{I}-\frac{1}{6} h^{I} \square h^{I}\right) .
\end{aligned}
$$

Combining Eqs. (A8), (A10), and (A12), we have the relation

$$
\begin{aligned}
L_{E} & \hat{h}_{\mu \nu}^{0}-\frac{1}{4} \nabla_{\rho} h^{I \rho \sigma} \nabla_{\mu} h_{\sigma \nu}^{I}-\frac{1}{4} \nabla_{\rho} h^{I \rho \sigma} \nabla_{\nu} h_{\sigma \mu}^{I}+\frac{1}{4} \nabla_{\rho} h^{I \rho \sigma} \nabla_{\sigma} h_{\mu \nu}^{I}+\frac{1}{8} \nabla_{\mu} h^{I \rho \sigma} \nabla_{\nu} h_{\rho \sigma}^{I}-\frac{1}{4} \nabla^{\sigma} h_{\rho \nu}^{I} \nabla^{\rho} h_{\sigma \mu}^{I}+\frac{1}{4} \nabla^{\sigma} h_{\nu}^{I \rho} \nabla_{\sigma} h_{\mu \rho}^{I} \\
& -\frac{1}{4} \nabla^{\rho} \nabla^{\sigma} h_{\rho \sigma}^{I} h_{\mu \nu}^{I}-\frac{1}{4} h^{I \rho \sigma} \nabla_{\rho} \nabla_{\mu} h_{\sigma \nu}^{I}-\frac{1}{4} h^{I \rho \sigma} \nabla_{\rho} \nabla_{\nu} h_{\sigma \mu}^{I}+\frac{1}{4} h^{I \rho \sigma} \nabla_{\rho} \nabla_{\sigma} h_{\mu \nu}^{I}+\frac{1}{4} h^{I \rho \sigma} \nabla_{\nu} \nabla_{\mu} h_{\rho \sigma}^{I}-\frac{\Lambda^{I}}{4} h_{\mu \rho}^{I} h_{\nu}^{I \rho} \\
& -\frac{1}{8} \nabla_{\rho} h_{\mu \nu}^{I} \nabla^{\rho} h^{I}+\frac{1}{8}\left(\nabla_{\mu} h_{\rho \nu}^{I}+\nabla_{\nu} h_{\rho \mu}^{I}\right) \nabla^{\rho} h^{I}+\frac{1}{4} h_{\mu \nu}^{I} \square h^{I}+\frac{3 \Lambda^{I}}{8} h_{\mu \nu}^{I} h^{I} \\
& +\frac{1}{8}\left(\nabla_{\mu} h_{\rho \nu}^{I}+\nabla_{\nu} h_{\rho \mu}^{I}\right) \nabla^{\rho} h^{I}-\frac{1}{8} \nabla_{\rho} h_{\mu \nu}^{I} \nabla^{\rho} h^{I}+\frac{1}{4} h_{\mu \nu}^{I} \square h^{I}+\frac{\Lambda^{I}}{8} h_{\mu \nu}^{I} h^{I}+\frac{1}{8} \nabla_{\mu} h^{I} \nabla_{\nu} h^{I}+\frac{1}{4} h^{I} \nabla_{\mu} \nabla_{\nu} h^{I} \\
& +g_{\mu \nu}\left(-\frac{1}{4} h^{I \rho \sigma} \nabla^{\tau} \nabla_{\rho} h_{\tau \sigma}^{I}+\frac{1}{8} h^{I \rho \sigma} \square h_{\rho \sigma}^{I}+\frac{3 \Lambda^{I}}{16} h^{I \rho \sigma} h_{\rho \sigma}^{I}+\frac{1}{8} h^{I \rho \sigma} \nabla_{\rho} \nabla_{\sigma} h^{I}\right. \\
& +\frac{1}{8} \nabla^{\rho} \nabla^{\sigma} h_{\rho \sigma}^{I} h^{I}-\frac{1}{8} h^{I} \square h^{I}-\frac{\Lambda^{I}}{8} h^{I} h^{I}+\frac{1}{8} \nabla_{\rho} h^{I \rho \sigma} \nabla_{\sigma} h^{I}+\frac{1}{4} h^{I \rho \sigma} \nabla_{\rho} \nabla_{\sigma} h^{I} \\
& \left.-\frac{1}{16} \nabla^{\rho} h^{I} \nabla_{\rho} h^{I}-\frac{1}{8} h^{I} \square h^{I}+\frac{\Lambda^{I}}{16} h^{I} h^{I}+\frac{1}{16} \nabla^{\rho} h^{I} \nabla_{\rho} h^{I}+\frac{1}{8} h^{I} \square h^{I}\right)=0,
\end{aligned}
$$

where $\hat{h}_{\mu \nu}^{0}=h_{\mu \nu}^{0}+\frac{1}{2} g_{\mu \nu} s^{0}$ is the effective four-dimensional graviton. 


\section{APPENDIX B: EFFECTIVE ENERGY- MOMENTUM TENSOR FROM GW PERTURBATION}

In this Appendix, we briefly review the four-dimensional energy-momentum tensor from GW perturbation, in order to show the analogy with what we did in Sec. III. In Sec. II, starting from the flat five-dimensional gravity theory and applying the KK reduction including terms that are of quadratic order in the metric perturbation, we have obtained the four-dimensional effective gravity theory with matter. A similar situation has been considered in four-dimensional gravity when a source of the GW is far from the observer. In this case, the energy carried by a wave as it leaves a source can be written as an effective stress-energy tensor for the wave by including the second-order perturbation. This is the so-called Isaacson energy-momentum tensor [51].

We assume that the amount of energy associated with the $\mathrm{GW}$ in a region of spacetime is large enough to contain several wavelengths of gravitational radiation but is smaller than any background curvature scale. In this case, one needs to find an integral average of an effective energymomentum tensor over a volume large enough that bulk contributions are greater than the boundary contributions. Einstein field equations in a vacuum are written as

$$
G_{\mu \nu}=\stackrel{0}{G}_{\mu \nu}+\stackrel{1}{G}_{\mu \nu}\left[h_{\alpha \beta}\right]+\stackrel{2}{G}_{\mu \nu}\left[h_{\alpha \beta}\right]+\cdots=0 .
$$

One can write the metric in the flat spacetime with its fluctuation as

$g_{\alpha \beta}=\eta_{\alpha \beta}+h_{\alpha \beta}=\eta_{\alpha \beta}+\lambda h_{\alpha \beta}+\lambda^{2} h_{\alpha \beta}^{2}+\cdots, \quad\left|h_{\alpha \beta}\right| \ll 1$,

where $\lambda$ is a formal order parameter denoting the first-order correction $(h)$ and the second-order correction $(h)$, respectively. Then, one can rewrite Eq. (B1) by using Eq. (B2) as

$$
\begin{aligned}
G_{\mu \nu}= & \stackrel{0}{G_{\mu \nu}}+\lambda \stackrel{1}{G}_{\mu \nu}\left[\frac{1}{h_{\alpha \beta}}\right]+\lambda^{2}\left(\stackrel{1}{G}_{\mu \nu}\left[2^{h} \alpha \beta\right]+\stackrel{2}{G}_{\mu \nu}\left[\frac{1}{h}{ }_{\alpha \beta}\right]\right) \\
& +\mathcal{O}\left(\lambda^{3}\right)+\cdots \\
= & 0 .
\end{aligned}
$$

The above equation should hold order by order in $\lambda$; then the first-order and second-order corrections to the background metric are given by [52]

$$
\begin{gathered}
\stackrel{0}{G}_{\mu \nu}=0, \\
\stackrel{1}{G}_{\mu \nu}\left[1_{\alpha \beta}\right]=0, \\
\stackrel{1}{G}_{\mu \nu}\left[\stackrel{2}{h}_{\alpha \beta}\right]=\stackrel{2}{G}_{\mu \nu}\left[h_{\alpha \beta}\right]=\frac{8 \pi G}{c^{4}} T_{\mu \nu}^{(\mathrm{GW})},
\end{gathered}
$$

where $T_{\mu \nu}^{(\mathrm{GW})} \equiv-\frac{c^{4}}{8 \pi G} \stackrel{2}{G}\left[\stackrel{1}{h}_{\alpha \beta}\right]$ is the effective energymomentum tensor created by the first-order GW perturbation. In order to make $T_{\mu \nu}^{(\mathrm{GW})}$ gauge invariant, one must perform an integral average over a region of spacetime large enough to contain several GW oscillations,

$$
T_{\mu \nu}^{\mathrm{GW}}=-\frac{c^{4}}{8 \pi G}\left\langle\stackrel{2}{R}_{\mu \nu}-\frac{1}{2} \eta_{\mu \nu} R\right\rangle,
$$

where \langle\rangle represents the integral average. To perform the above calculation, one uses the definition of the Ricci tensor, $R_{\mu \nu}=\frac{\partial \Gamma_{\mu \nu}^{\alpha}}{\partial x^{\alpha}}-\frac{\partial \Gamma_{\alpha \nu}^{\alpha}}{\partial x^{\mu}}+\Gamma_{\mu \nu}^{\alpha} \Gamma_{\alpha \beta}^{\beta}-\Gamma_{\beta \nu}^{\alpha} \Gamma_{\mu \alpha}^{\beta}$, and retains the terms quadratic in $h_{\mu \nu}$ while applying the following tricks: (i) adopt the TT gauge so that $h_{0 \mu}^{\mathrm{TT}}=0, \delta^{i k} \partial h_{i j}^{\mathrm{TT}} / \partial x^{k}=0$, and $\delta^{i j} h_{i j}^{\mathrm{TT}}=0$; (ii) assume that under an integral average, all terms of the form $\left\langle\partial T_{\mu \cdots \gamma} / \partial x^{\nu}\right\rangle$ can be neglected since such terms contribute only to the boundary of the region and can be made arbitrarily small compared to the bulk by expanding the region in which the integral average is performed; and (iii) use $\square h_{i j}^{\mathrm{TT}}=0$.
[1] B. P. Abbott et al. (LIGO Scientific and Virgo Collaborations), Observation of Gravitational Waves from a Binary Black Hole Merger, Phys. Rev. Lett. 116, 061102 (2016).

[2] T. D. Abbott et al. (LIGO Scientific and Virgo Collaborations), Improved Analysis of GW150914 Using a Fully Spin-Precessing Waveform Model, Phys. Rev. X 6, 041014 (2016).

[3] B. P. Abbott et al. (LIGO Scientific and Virgo Collaborations), GW151226: Observation of Gravitational Waves from a 22-Solar-Mass Binary Black Hole Coalescence, Phys. Rev. Lett. 116, 241103 (2016).
[4] B. P. Abbott et al. (LIGO Scientific and Virgo Collaborations), Binary Black Hole Mergers in the first Advanced LIGO Observing Run, Phys. Rev. X 6, 041015 (2016); Errtaum, Phys. Rev. X 8, 039903(E) (2018).

[5] B. P. Abbott et al. (LIGO Scientific and Virgo Collaborations), GW170104: Observation of a 50-Solar-Mass Binary Black Hole Coalescence at Redshift 0.2, Phys. Rev. Lett. 118, 221101 (2017); Errtaum, Phys. Rev. Lett. 121, 129901(E) (2018).

[6] B. P. Abbott et al. (LIGO Scientific and Virgo Collaborations), GW170814: A Three-Detector Observation 
of Gravitational Waves from a Binary Black Hole Coalescence, Phys. Rev. Lett. 119, 141101 (2017).

[7] B. P. Abbott et al. (LIGO Scientific and Virgo Collaborations), GW170608: Observation of a 19-solar-mass binary black hole coalescence, Astrophys. J. 851, L35 (2017).

[8] A. H. Nitz, C. Capano, A. B. Nielsen, S. Reyes, R. White, D. A. Brown, and B. Krishnan, 1-OGC: The first open gravitational-wave catalog of binary mergers from analysis of public Advanced LIGO data, Astrophys. J. 872, 195 (2019).

[9] B. P. Abbott et al. (LIGO Scientific and Virgo Collaborations), GWTC-1: A Gravitational-Wave Transient Catalog of Compact Binary Mergers Observed by LIGO and Virgo during the First and Second Observing Runs, Phys. Rev. X 9, 031040 (2019).

[10] B. P. Abbott et al. (LIGO Scientific and Virgo Collaborations), GW170817: Observation of Gravitational Waves from a Binary Neutron Star Inspiral, Phys. Rev. Lett. 119, 161101 (2017).

[11] B. P. Abbott et al. Multi-messenger observations of a binary neutron star merger, Astrophys. J. 848, L12 (2017).

[12] G. Nordström, Über die Möglichkeit, das elektromagnetische Feld und das Gravitationsfeld zu vereinigen, Phys. Z. 15, 504 (1914).

[13] T. Kaluza, Zum Unitätsproblem der Physik, Sitzungsberichte der Königlich Preußischen Akademie der Wissenschaften (Berlin), Seite (1921).

[14] O. Klein, Quantentheorie und fünfdimensionale Relativitätstheorie, Z. Phys. 37, 895 (1926).

[15] E. Witten, Search for a realistic Kaluza-Klein theory, Nucl. Phys. B186, 412 (1981).

[16] J. M. Overduin and P. S. Wesson, Kaluza-Klein gravity, Phys. Rep. 283, 303 (1997).

[17] A. O. Barvinsky and S. N. Solodukhin, Echoing the extra dimension, Nucl. Phys. B675, 159 (2003).

[18] V. Cardoso, O. J. C. Dias, and J. P. S. Lemos, Gravitational radiation in D-dimensional space-times, Phys. Rev. D 67, 064026 (2003).

[19] V. Cardoso, S. Yoshida, O. J. C. Dias, and J. P. S. Lemos, Late time tails of wave propagation in higher dimensional space-times, Phys. Rev. D 68, 061503 (2003).

[20] V. Cardoso, O. J. C. Dias, and P. Figueras, Gravitational radiation in $d>4$ from effective field theory, Phys. Rev. D 78, 105010 (2008).

[21] E. Alesci and G. Montani, Can be gravitational waves markers for an extra-dimension?, Int. J. Mod. Phys. D 14, 923 (2005).

[22] H. Yu, B. M. Gu, F. P. Huang, Y. Q. Wang, X. H. Meng, and Y.X. Liu, Probing extra dimension through gravitational wave observations of compact binaries and their electromagnetic counterparts, J. Cosmol. Astropart. Phys. 02 (2017) 039.

[23] D. Andriot and G. L. Gómez, Signatures of extra dimensions in gravitational waves, J. Cosmol. Astropart. Phys. 06 (2017) 048.

[24] S. Chakraborty, K. Chakravarti, S. Bose, and S. SenGupta, Signatures of extra dimensions in gravitational waves from black hole quasinormal modes, Phys. Rev. D 97, 104053 (2018).

[25] L. Visinelli, N. Bolis, and S. Vagnozzi, Brane-world extra dimensions in light of GW170817, Phys. Rev. D 97, 064039 (2018).
[26] K. Pardo, M. Fishbach, D. E. Holz, and D. N. Spergel, Limits on the number of spacetime dimensions from GW170817, J. Cosmol. Astropart. Phys. 07 (2018) 048.

[27] E. McDonough and S. Alexander, Observable chiral gravitational waves from inflation in string theory, J. Cosmol. Astropart. Phys. 11 (2018) 030.

[28] To provide a physical interpretation for the Kaluza's cylinder condition [13], Klein considered the same setting in the $L \rightarrow 0$ limit [14]. So the information of the extra dimension is not encoded in this reduction procedure.

[29] One can naturally extend our setting to the case of the $(4+n)$-dimensional spacetime with $n$-dimensional extra dimensions with a Ricci flat background. Then, the background manifold becomes $\mathcal{M}_{4} \times T^{n}$, where $T^{n}$ represents the $n$-torus. Since there will be no fundamental difference from the case of the five-dimensional setting, we concentrate on the five-dimensional case, for simplicity.

[30] We denote the five-dimensional spacetime indices with the Roman letters $p, q, \ldots$, and the four-dimensional ones with the Greek letters $\mu, \nu, \ldots$.

[31] From now on, we drop $\sum_{I=0}^{\infty}$ and use the Einstein summation convention, for simplicity.

[32] M. Maggiore, Gravitational wave experiments and early universe cosmology, Phys. Rep. 331, 283 (2000).

[33] M. Maggiore, Gravitational Waves, Vol. 1: Theory and Experiments (Oxford University Press, Oxford, 2008).

[34] J. W. Armstrong, L. Iess, P. Tortora, and B. Bertotti, Stochastic gravitational wave background: Upper limits in the $10^{6}$ to $10^{3} \mathrm{~Hz}$ band, Astrophys. J. 599, 806 (2003).

[35] A. Shoda, M. Ando, K. Ishidoshiro, K. Okada, W. Kokuyama, Y. Aso, and K. Tsubono, Search for a stochastic gravitational-wave background using a pair of torsion-bar antennas, Phys. Rev. D 89, 027101 (2014).

[36] M. Coughlin and J. Harms, Upper Limit on a Stochastic Background of Gravitational Waves from Seismic Measurements in the Range $0.051 \mathrm{~Hz}$, Phys. Rev. Lett. 112, 101102 (2014).

[37] M. Coughlin and J. Harms, Constraining the gravitational wave energy density of the Universe using Earths ring, Phys. Rev. D 90, 042005 (2014).

[38] J. Aasi et al. (LIGO Scientific and Virgo Collaborations), Improved Upper Limits on the Stochastic GravitationalWave Background from 20092010 LIGO and Virgo Data, Phys. Rev. Lett. 113, 231101 (2014).

[39] M. Coughlin and J. Harms, Constraining the gravitationalwave energy density of the Universe in the range $0.1 \mathrm{~Hz}$ to $1 \mathrm{~Hz}$ using the apollo seismic array, Phys. Rev. D 90, 102001 (2014).

[40] R. Adam et al. (Planck Collaboration), Planck intermediate results. XXX. The angular power spectrum of polarized dust emission at intermediate and high Galactic latitudes, Astron. Astrophys. 586, A133 (2016).

[41] J. Scherk and J. H. Schwarz, How to get masses from extra dimensions, Nucl. Phys. B153, 61 (1979).

[42] See Appendix A for the details.

[43] M. Tanabashi, K. Hagiwara, K. Hikasa et al., Review of particle physics, Phys. Rev. D 98, 030001 (2018).

[44] L. Randall and R. Sundrum, A Large Mass Hierarchy from a Small Extra Dimension, Phys. Rev. Lett. 83, 3370 (1999). 
[45] C. Csaki, M. L. Graesser, and G. D. Kribs, Radion dynamics and electroweak physics, Phys. Rev. D 63, 065002 (2001).

[46] T. Tanaka and X. Montes, Gravity in the brane world for two-branes model with stabilized modulus, Nucl. Phys. B582, 259 (2000).

[47] M. Frank, K. Huitu, U. Maitra, and M. Patra, Probing Higgs-radion mixing in warped models through complementary searches at the LHC and the ILC, Phys. Rev. D 94, 055016 (2016).

[48] L. Dolan and M. J. Duff, Kac-Moody Symmetries of Kaluza-Klein Theories, Phys. Rev. Lett. 52, 14 (1984).
[49] Y. M. Cho and S. W. Zoh, Explicit construction of massive spin-two fields in Kaluza-Klein theory, Phys. Rev. D 46, R2290 (1992).

[50] Y. M. Cho and S. W. Zoh, Virasoro invariance and theory of internal string, Phys. Rev. D 46, 3483 (1992).

[51] R. Isaacson, Gravitational radiation in the limit of high frequency. II. Nonlinear terms and the effective stress tensor, Phys. Rev. 166, 1272 (1968).

[52] C. W. Misner, K. S. Thorne, and J. A. Wheeler, Gravitation (Princeton University Press, Princeton, NJ, 1973). 